\title{
The celestial mechanics approach: theoretical foundations
}

\author{
Gerhard Beutler • Adrian Jäggi · Leoš Mervart • \\ Ulrich Meyer
}

Received: 31 October 2009 / Accepted: 29 July 2010 / Published online: 24 August 2010

(c) Springer-Verlag 2010

\begin{abstract}
Gravity field determination using the measurements of Global Positioning receivers onboard low Earth orbiters and inter-satellite measurements in a constellation of satellites is a generalized orbit determination problem involving all satellites of the constellation. The celestial mechanics approach (CMA) is comprehensive in the sense that it encompasses many different methods currently in use, in particular so-called short-arc methods, reduced-dynamic methods, and pure dynamic methods. The method is very flexible because the actual solution type may be selected just prior to the combination of the satellite-, arc- and technique-specific normal equation systems. It is thus possible to generate ensembles of substantially different solutions-essentially at the cost of generating one particular solution. The article outlines the general aspects of orbit and gravity field determination. Then the focus is put on the particularities of the CMA, in particular on the way to use accelerometer data and the statistical information associated with it.
\end{abstract}

Keywords Celestial mechanics - Orbit determination · Global gravity field modeling · CHAMP · GRACE

\section{Problem description and overview}

This article has the focus on the theoretical foundations of the so-called celestial mechanics approach (CMA). Applications

G. Beutler $(\bowtie)$ · A. Jäggi · U. Meyer

Astronomical Institute, University of Bern, Sidlerstrasse 5, 3012 Bern, Switzerland

e-mail: gerhard.beutler@aiub.unibe.ch

\section{Mervart}

Institute of Advanced Geodesy, Czech Technical University, K-152, FSvCVVT, Thakurova 7, 16629 Prague 6-Dejvice,

Czech Republic of the CMA, in particular to the GRACE mission, may be found in Beutler et al. (2010) and Jäggi et al. (2010b).

The determination of the Earth's global gravity field using the data of space missions is nowadays either based on

1. the observations of spaceborne Global Positioning (GPS) receivers onboard low Earth orbiters (LEOs) (see Reigber et al. 2004),

2. or precise inter-satellite distance monitoring of a close satellite constellation using microwave links (combined with the measurements of the GPS receivers on all spacecrafts involved) (see Tapley et al. 2004),

3. or on gradiometer measurements realized by three pairs of three-dimensional accelerometers onboard a LEO measuring the complete gravitational tensor of the Earth along the satellite's trajectory (combined with the observations of the onboard GPS receiver) (see Drinkwater et al. 2006).

The CHAllenging Minisatellite Payload (CHAMP) mission is generating the first kind of data set, the Gravity Recovery And Climate Experiment (GRACE) mission the second one, and the Gravity field and steady-state Ocean Circulation Explorer (GOCE) mission the third one. The first type of data is available for all three missions.

The gravity fields emerging from one of the above data sets are often combined in a statistically correct way with the solutions obtained from Satellite Laser Ranging (SLR) and/or from ground-based or airborne gravimetry (see Förste et al. 2008). We do not consider combinations of this kind here, but focus on the contribution of the mentioned satellite missions.

The above observational data are supported by the measurements of accelerometers (Touboul et al. 1999), e.g., placed in the satellite's center of mass, which give-as a 
function of time-biased and scaled values of the nongravitational accelerations acting on the satellites. The measurements are performed in three orthogonal directions, usually closely related to the radial $(R)$, the out-of-plane $(W)$, and the along-track component $(S)$, which are associated with the unit vectors $\mathbf{e}_{r}$ in the radial, $\mathbf{e}_{W}$ in the out-of-plane, and $\mathbf{e}_{S} \doteq \mathbf{e}_{W} \times \mathbf{e}_{R}$ in the (approximate) along-track directions. Accelerometer bias parameters have to be set up and estimated in the generalized orbit estimation process. One should also solve for accelerometer scale parameters. These parameters are, however, highly correlated with the onceper-revolution dynamic parameters, which is why we usually do not solve for them. In order to refer the GPS- and inter-satellite measurements to the satellites' centers of mass, the attitude of the satellite-fixed coordinate system has to be oriented in the inertial system (using star-tracking cameras) and the sensor offsets in the satellite-fixed coordinate system have to be known.

The complexity of the parameter estimation problem may be considerably reduced by not analyzing the original (phase and code) GPS measurements in the gravity field estimation process, but rather the LEOs' so-called kinematic positions (Švehla et al. 2004), using optionally parts of the variancecovariance matrices, hereafter called simply covariance matrices, associated with them. As the GPS-derived kinematic positions are not original measurements, but used subsequently as observations in the parameter estimation procedures, we also refer to them as pseudo-observations or pseudo-measurements. Kinematic LEO orbits are derived from the LEO receivers' GPS code and phase measurements using a precise point positioning (PPP) procedure (Zumberge et al. 1997). More information is provided in Sect. 3.2.

Static "satellite-only" gravity fields based on long data spans should be based on solutions not making use of information other than that contained in the measurements. Combinations with other solutions on the normal equation system (NEQ) level involving other techniques should be made after the satellite-only solutions. This demand is "rather absolute" and never can be really met, because one will, e.g., always make use (and be it only for reasons of computation efficiency) of a rather good a priori gravity field - meaning that reasonable approximate values are used at least for the terms up to degree $n=20$. The dependency on the a priori gravity field was studied in some detail in Jäggi et al. (2010b), where the EGM96 (Lemoine et al. 1997) served as a priori field. Virtually the same results were obtained when using a much better a priori field. We are therefore confident that the CMA meets the demand of "independency on the a priori gravity field" to a great extent. As long as all coefficients set up in the a priori field are also estimated in the subsequent parameter estimation procedure, the impact of the a priori gravity field on the estimated field must be small—and should disappear completely, if the solution is iteratively improved using the estimated parameters as new a priori values in a second iteration step. The dependency on many other background models (e.g., tides) are much more problematic and difficult to avoid.

The situation is different if the interest is on the time varying part of the gravity field using a rather short data span (usually one month in the case of GRACE). There it may make sense to generate solutions, which are based on an a priori gravity field of high degree and order (e.g., with a cutoff degree of $n=150-180$, which was derived previously), and to determine the gravity field parameters up to a modest limiting degree only, e.g., between 20 and 60 . The aspect of extracting the time varying part of the gravity field is, however, not in the center of this article. Such a set-up to study the impact of different solution strategies on the achievable results is used in Beutler et al. (2010). Note, as well, that several research centers determine monthly gravity fields from GRACE up to degree 120 and that Liu et al. (2010) retrieved temporal signals above degree 70 in monthly GRACE gravity fields using filtering techniques.

The CMA has its roots in the Bernese GPS Software (Dach et al. 2007), which was extensively used for determining the orbits of Global Navigation Satellite Systems (GNSS). The CMA was generalized to determine the orbits of LEOs by Jäggi (2007) with special emphasis on the stochastic properties of the orbits. In recent years the CMA was further developed and used for gravity field determination. This article represents the first consistent description of the CMA, the key aspects of which are:

- The CMA is based on the foundations of celestial mechanics. It is a package designed for orbit and for gravity field determination, where the latter task is merely a generalization of the former.

- The modularity of the CMA allows it to study or improve individual contributions to a resulting orbit or gravity field. It also may be used as an ideal tool for planning future space missions (gravity field oriented or other).

- The use of kinematic positions derived from GPS, together with the options to use either the full or only the epoch-specific covariance matrices from PPP, is perfectly suited to study the quality of GPS-only orbits and their contribution to gravity fields.

- The CMA is based not on stochastic, but on piecewise deterministic equations of motion. With its capabilities to set up pseudo-stochastic parameters (of different kinds) with a dense spacing, the CMA orbits may be made close relatives of solutions of stochastic differential equations.

The aspects of Celestial Mechanics relevant for gravity field determination are described in Sect. 2. The CMA's response to these challenges may be found in Sect. 3. Section 4 reviews different methods used for gravity field determination and 
puts them in relation to the CMA. Section 5 summarizes the main findings and the conclusions emerging from this work.

\section{Orbit and gravity field determination}

Every procedure to determine the Earth's gravity field using space data has to be based on the foundations of celestial mechanics. A static gravity field to be derived from the GOCE mission seems to be an exception at first sight, because the key instrument, the gradiometer, provides the gravitational tensor along the satellite's trajectories as in situ measurements, which depend only weakly on the satellite's orbital motion. Due to the limited bandwidth of the gradiometer, however, the lower degree harmonics are almost uniquely determined by the orbital motion of the GOCE satellite (Pail et al. 2006). Because of the low altitude of the satellite, the gravity field derived from the GOCE GPS data alone should already be of remarkable quality and resolution-and it has to be combined in a consistent way with the results emerging from the gradiometer.

Our procedure should be called more precisely "the method to generate ensembles of orbits and gravity field solutions fully exploiting the degrees of freedom offered by celestial mechanics and using the power of applied mathematics to generate the precise solutions in an efficient way". As this description is of the lengthy kind, we stick to the short title "CMA".

Before highlighting the essential elements of our method in Sect. 3 we review the key characteristics of orbital motion and of gravity field determination in general in this section.

\subsection{Equations of motion and their solution}

The geocentric position vector $\mathbf{r}(t)$ of a satellite's center of mass solves the so-called equations of motion for each time argument $t$. These are usually written as second order, non-linear ordinary differential equations based on Newton's principles and on his law of universal gravitation, amended by corrections due to the special and general theories of relativity. The equations used today are often referred to as parameterized post-Newtonian equations (Seidelmann 1992). The satellite's position vector $\mathbf{r}(t)$ at any given epoch $t$ is a particular solution of the equations of motion defined by the initial position and velocity vectors $\mathbf{r}_{0} \doteq \mathbf{r}\left(t_{0}\right)$ and $\mathbf{v}_{0} \doteq \dot{\mathbf{r}}\left(t_{0}\right)$ at an initial epoch $t_{0}$. The initial position and velocity vectors are also referred to as "initial state vector" (with six elements). The initial state vector is defined by six quantities, e.g., the initial osculating orbital elements. For a general discussion of the orbit determination problem see Beutler (2005, Vol. 1, Chapter 8).

In order to solve the parameter estimation problem, the relationship between the observables (see Sect. 2.2) and the parameters has to be linearized. Linearization implies for our problems that each orbit (arc) involved is approximated as a linear function of its defining parameters including those dynamical parameters common to several or all orbits. In a generic way a linearized orbit may be written as

$\mathbf{r}(t) \doteq \mathbf{r}_{0}(t)+\sum_{i=1}^{n_{\mathrm{par}}} \frac{\partial \mathbf{r}_{0}(t)}{\partial p_{i}}\left(p_{i}-p_{0 i}\right)$,

where the a priori orbit (or "initial orbit" or "reference orbit") $\mathbf{r}_{0}(t) \doteq \mathbf{r}\left(t ; p_{01}, p_{02}, \ldots, p_{0, n_{\text {par }}}\right)$ is a function of time and characterized by known approximate values $p_{0 i}$ of the parameters $p_{i}, i=1,2, \ldots, n_{\mathrm{par}}$; the partial derivatives on the righthand side should be understood as the partial derivatives of the function $\mathbf{r}\left(t ; p_{1}, p_{2}, \ldots, p_{n_{\text {par }}}\right)$ w.r.t. the parameters $p_{i}$, evaluated at $p_{i}=p_{0 i} ; n_{\mathrm{par}}$ is the number of orbit parameters defining the orbit $\mathbf{r}(t)$. Whereas all methods are based on a linearized representation of the unknown orbits as a function of the parameters, one should clearly specify how the reference orbits and the partial derivatives of the reference orbits w.r.t. the parameters of different type are generated.

\subsection{Observables and observations}

Two classes of observations (measurements) should be distinguished in modern gravity field determination:

- Class I: observations measuring functions of the satellites' position and/or velocity vectors at particular epochs,

- Class II: observations measuring (parts of) the force field (or functions of the field) acting on the satellites at particular epochs.

The observed functions of the parameters are also referred to as observables.

Kinematic positions derived from spaceborne GPS receivers and the K-Band range or range-rate Level $1 \mathrm{~b}$ measurements of the GRACE mission are typical representatives of observations of Class I, accelerometer and gradiometer measurements of Class II.

The observations actually used in the parameter adjustment process may be the original measurements gained by the satellites' sensors or functions thereof. Often it is assumed that the errors in the original measurements (e.g., the GPS phase or the K-Band Level 1a range measurements) have particularly simple statistical characteristics, e.g., independent and normally distributed. It does not matter whether the original measurements or functions thereof are analyzed in the adjustment process-as long as the mathematical correlations between the errors in the original measurements and those analyzed are taken into account. If, e.g., the full covariance matrix of the kinematic positions covering the entire 
time span of data as a function of the GPS phase (and code) observations is available and used in the adjustment process (what is, however, usually not done), the results are the same as if the orbits and/or the gravity fields would have been estimated directly with the original observations. The same remarks apply to the use of Level $1 \mathrm{~b}$ ranges or range-rates instead of the Level 1a ranges.

In the absence of Class II observations parameter estimation is a straightforward affair. The observations are either independent with known variances or they are linear combinations of the original observations of Class I. In the former case the weight matrix is diagonal, in the latter case fully populated. The impact of the non-modeled parts of the force field are treated as stochastic quantities by the adjustment process, reflected by an enlarged root mean square (RMS) error a posteriori (w.r.t. the known RMS error a priori) of the Class I observations. Pure dynamic methods are usually based on this procedure.

Properly taking into account the stochastic properties of Class II observations is in practice less trivial, because the observables of Class I are functions of the force field, as well, i.e., also of the observations of Class II. It is, however, in principle easy to set up a correct analysis involving both classes of observations:

1. The observations of the force field have to be introduced with their known statistical properties into the adjustment process, i.e., in addition to the Class I observation equations there is also one observation equation for each measurement of Class II.

2. The impact of the observables of Class II on the observations of Class I has to be taken into account.

Let us outline the correct procedure using the Level 1a accelerometer observations $a_{k}^{\prime}$ of the GRACE mission as an example. We assume that these measurements are independent and of the same accuracy. In practice, the measurements $a_{k}^{\prime}$ are filtered, resulting in a smoothed series $a_{k 0}$, which is then used as the empirically given non-gravitational force in the integration process. In order to analyze the structure of the problem we skip the filtering process and assume that the empirical non-gravitational forces are constant in the time intervals $\left[t_{k}, t_{k+1}\right]$ and directly given by the Level 1a data

$a_{k 0} \doteq a_{k}^{\prime}, \quad t \in\left[t_{k}, t_{k+1}\right], \quad k=1,2, \ldots$

This view is statistically correct, because the use of the unfiltered data is equivalent to the use of the filtered data together with the corresponding correct correlation matrix.

In order to take the statistical nature of the measurements $a_{k}^{\prime}$ into account one must introduce the parameters $\Delta a_{k}$, standing for the difference between the true and the measured acceleration. The measurements thus may be written as $a_{k}^{\prime} \doteq a_{k 0}-\Delta a_{k}$. The quantities $\Delta a_{k}$ are normal parameters of the adjustment process governed by the observation equation

$\Delta a_{k}=0, \quad k=1,2, \ldots$

Assuming that the measurements $a_{k}^{\prime}$ are not correlated and all have the same variance $\sigma_{a}^{2}$, the above measurements are associated with the weights $\sigma_{0}^{2} / \sigma_{a}^{2}$, where $\sigma_{0}$ is the standard deviation of the weight unit used in the adjustment.

The impact of the parameters $\Delta a_{k}$ on the kinematic positions and inter-satellite distances has to be taken into account, as well. This problem is, in principle, easily solved: the dependency of the orbit on the parameters is described in a generic way by Eq. (1), where the $\Delta a_{k}$ are elements of the parameter list. The position and/or velocity vectors are therefore also linear functions of the $\Delta a_{k}$ corresponding to epochs prior to the observation epoch. The same linear dependency thus also holds for functions of these vectors.

The partial derivatives w.r.t. the $\Delta a_{k}$ can be calculated, without additional computational burden, as linear combinations of the partial derivatives w.r.t. the six parameters defining the initial state vector and the partial derivative w.r.t. a constant force in the direction considered. As the functional dependency of the Class I observables on the satellite positions and velocities is known anyway for each time argument $t$, it is also straightforward to model the influence of these new parameters $\Delta a_{k}$ on the Class I observables like range, range-rate, etc. This completes the correct way of taking the stochastic properties of the accelerometer measurements into account. No problems of principle have been encountered.

The problem with this purist approach resides in the potentially huge number of additional parameters associated with it: when using accelerometer data with a $0.1 \mathrm{~s}$ spacing, one ends up with 864,000 additional parameters in the daily NEQs - definitely a hopeless affair. The problem can of course be alleviated by taking into account the correlations only over short time periods and by (pre-)eliminating the parameters $\Delta a_{k}$ of the short time intervals. There are ways to do this efficiently.

The effort of taking into account the impact of Class II on Class I observables becomes irrelevant, if their impact on the Class I observables is much smaller than the Class I RMS errors. For GRACE-like missions this requirement obviously would have an impact on the stochastic properties of the range and of the accelerometer observables.

\subsection{An experiment applied to an ideal gravity mission}

The following simulation illustrates that the above postulated independence is not given for all observables of the GRACE mission: a Keplerian orbit is perturbed by normally distributed, random accelerations $a_{R, k}^{\prime}, a_{S, k}^{\prime}, a_{W, k}^{\prime}$ in the $R$-, $S$ - and $W$-directions, which are constant in the time intervals $\left[t_{k}, t_{k+1}\right], k=0,1,2, \ldots$, where $t_{k+1}-t_{k}=\Delta t_{p}=$ 
Fig. 1 Residuals in $R$-, $S$-, $W$-directions of an orbit perturbed by piecewise constant accelerations (spacing of $0.1 \mathrm{~s}$, RMS $=10^{-10} \mathrm{~m} / \mathrm{s}^{2}$ ) using velocities (left) or positions (right) as observations, both without measurement noise
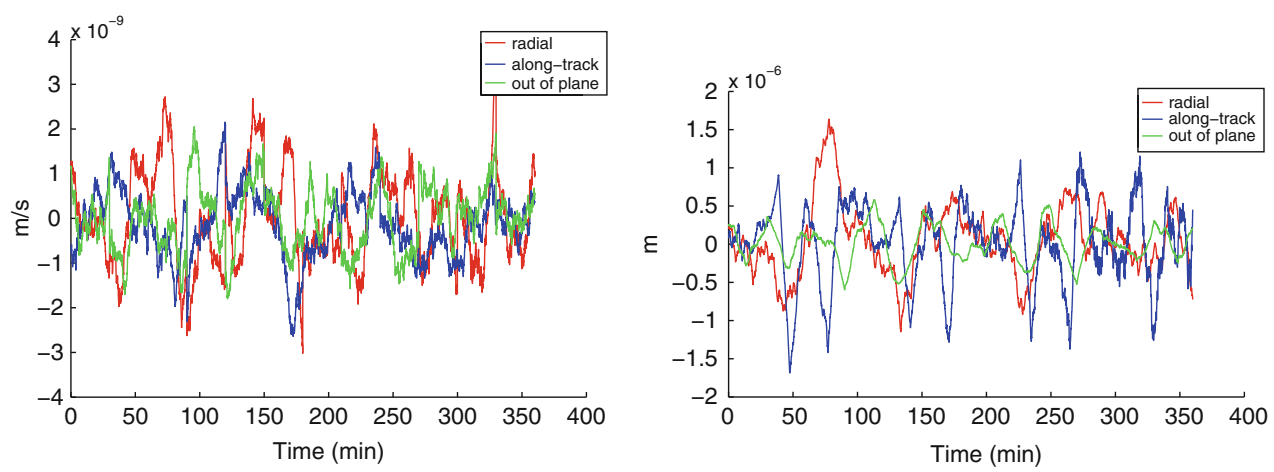

$0.1 \mathrm{~s}$. An RMS error of $\sigma_{a}=10^{-10} \mathrm{~m} / \mathrm{s}^{2}$ is assumed for these normally distributed piecewise constant accelerations in the three directions.

The components of the perturbed position or velocity vector are used as observations (without adding any additional measurement noise) in a conventional least-squares process, which assumes all Class 1 observables as independent and of the same accuracy (weight matrix = identity matrix), to estimate the orbit of a $6 \mathrm{~h}$-arc. The simulation represents a hypothetical "super gravity field experiment", which measures each component of the two satellites' position or velocity vectors with infinite accuracy. Its only "weakness" resides in the limited accuracy of the accelerometers. The six initial osculating elements and the unconstrained pulses in the $R$-, $S$ - and $W$-directions at 30-min intervals are the parameters of the orbit determination procedure. Figure 1 (left) shows the residuals of the orbit determination experiment using the velocity components as observations, and Fig. 1 (right) the residuals using the position components. Despite the fact that the measurement noise of the components of the velocity and position vectors was assumed to be zero in our experiment, the RMS error a posteriori of the orbit determination (based, as mentioned, on a conventional least-squares estimator) using the components of the velocity vector as observations is of the order of $10^{-9} \mathrm{~m} / \mathrm{s}$, it is of the order of $10^{-6} \mathrm{~m}$ when using the components of the position vector. Whether or not this accelerometer-induced noise on the Class I observables can be ignored depends on the noise characteristics of the Class I observables.

The results of the simulation experiment may be translated, to some extent, to the realities of the GRACE mission: the GRACE accelerometer measurements-when performing at the promised nominal level-have an impact of the kind illustrated by Fig. 1 on the components of the velocity and the position vector, respectively. These small effects do not matter at all when considering the impact on the GPSderived positions (we are speaking of position RMS errors of few centimeters for the kinematic positions).

The accelerometers also have an impact of the order of magnitude given by Fig. 1 (multiplied by $\sqrt{2}$ ) on the inter-satellite range-rates and ranges. The RMS errors of the order of $1-2 \times 10^{-7} \mathrm{~m} / \mathrm{s}$ for inter-satellite range-rates using a K-Band link (derived by numerical differentiation from ranges) between the satellites is still large compared to the accelerometer-induced noise in Fig. 1 (left). We therefore conclude that the impact of the stochastic properties of the GRACE accelerometers on the velocity observable is negligible in terms of the RMS estimated by the least-squares adjustment-provided the accelerometers perform as claimed by Touboul et al. (1999). The situation is not so clear for the range observable: Fig. 1 (right) indicates that small accelerometer RMS errors of $\sigma_{a} \approx 10^{-10} \mathrm{~m} / \mathrm{s}^{2}$ generate a systematic pattern in the range residuals of the order of $10^{-6} \mathrm{~m}$. The impact of the accelerometer-induced noise on the range observable is thus of the same order of magnitude as the RMS of the Level 1b range measurements and should be captured by methods described in the previous section.

The experiment performed in this section was based, as stated initially, on simplifying assumptions. An in-depth analysis should not be based simply on a comparison of RMS values of the involved observations, because of a possible dependency of noise on frequency, but on the power spectral densities of the range (or range-rate), the accelerometer, and the GPS-related observables.

\subsection{Primary and variational equations}

The initial orbit $\mathbf{r}_{0}(t)$ in Eq. (1) should be a sufficiently good approximation of the "final" orbit $\mathbf{r}(t)$ (resulting in the parameter estimation process) in order to justify the negligence of the non-linear terms.

The initial orbit $\mathbf{r}_{0}(t)$ is usually obtained by numerically solving the equations of motion. The partial derivatives in Eq. (1) solve the so-called variational equations (see Sect. 3.7). As the number of parameters is counted in tens of thousands in gravity field determination, it is important to solve the variational equations efficiently.

In the context of parameter estimation the equations of motion are called the primary equations. The primary equations have to be solved as accurately as needed, the actual 
requirements being dictated by the accuracy of the observations. When analyzing inter-satellite distances accurate to a few microns, "as accurately as needed" translates into "as accurately as possible" (see also Sect. 3.5).

\subsection{Parameter estimation}

Gravity field determination only makes sense, if the total number of observations vastly exceeds the total number of parameters. An estimation principle has to transform the observation equations into a system of equations, where the dimension equals the number of parameters. The method of least-squares demands the sum of the weighted residual squares to be minimum, which in turn implies that the partial derivatives of this sum w.r.t. each parameter are zero. The least-squares principle thus leads to a system of linear equations, the NEQs, the dimension of which equals the number of parameters. For more information concerning least-squares and other estimation principles we refer to Strang and Borre (1997).

\subsection{Arc, arc length}

We refer to a satellite arc as the trajectory $\mathbf{r}(t)$ between time limits $t_{b} \leq t \leq t_{e}$, represented by one and the same initial position and velocity vectors $\mathbf{r}_{0} \doteq \mathbf{r}\left(t_{0}\right)$ and $\mathbf{v}_{0} \doteq \dot{\mathbf{r}}\left(t_{0}\right)$. The arc length is the length $\left|t_{e}-t_{b}\right|$ of the time interval $I_{\text {arc }}=\left[t_{b}, t_{e}\right]$. Usually this time interval coincides with the interval containing all observations used to determine the initial state vector and other orbit parameters. $t_{0}$ usually coincides with $t_{b}$.

The arc length is an important option available to the analyst. In the history of celestial mechanics one usually made the attempt to render the arcs as long as possible in order to minimize the total number of parameters of a particular task (Biancale et al. 2000). Prior to the satellite missions of the twenty-first century most classical problems were governed by relatively few observations.

The age of modern gravity field determination has led to a revision of this concept. The availability of virtually continuous tracking by GPS (typically allowing for the determination of a satellite position every 10-30 s) and of continuous intersatellite measurements made short-arc methods a valuable and attractive alternative absorbing many biases of unclear origin into the initial state vectors. The success of procedures developed by Mayer-Gürr et al. (2005) and Mayer-Gürr (2008) underlines this statement.

\subsection{Orbit determination}

Orbit determination was originally understood as the task of determining six parameters, defining the initial state vector of the satellite, using all observations available in a certain time interval. A slightly more general case is that of determining the initial state vector and a certain number of arc-specific parameters from the mentioned set of observations (see, e.g., Beutler 2005). In the most general case one has to determine in addition parameters defining the force field. Such parameters may be either satellite- and arc-specific or -independent. Parameters of this kind may be called dynamic parameters. As gravity field parameters are dynamic parameters, gravity field determination is nothing but a generalized orbit determination task.

As the same dynamic parameters may show up in orbits of different satellites or in different arcs of the same satellite, the generalized orbit determination problem may be more complex than the pure orbit determination problem: the arcspecific parameters have to be pre-eliminated from the arcspecific NEQs, these reduced NEQs have to be accumulated and eventually the dynamic parameters common to all arcs and satellites have to be estimated by solving the resulting NEQ. The arc-specific parameters may then be calculated in a re-substitution procedure (if sufficient information was stored in the pre-elimination step) or by a pure orbit determination problem using the previously determined satelliteand arc-independent parameters as known values.

\subsection{Empirical and non-gravitational accelerations}

From the mathematical point of view empirical dynamic forces are indistinguishable from deterministic forces based on a physical force model. Empirical dynamic forces usually are meant to absorb poorly modelled parts of the force field. Typical empirical forces are constant, or once-, twice-, etc. per-revolution forces in predefined directions (e.g., in $R, S$, and $W$ ). The independent argument associated with the periodic forces needs to be specified (e.g., the argument of latitude $u$, the osculating true anomaly $v$, etc.).

Empirical forces are, e.g., required to solve for at least three constant accelerations per arc (in the 3 nominally orthogonal measurement directions), when using threedimensional accelerometers to define the non-gravitational forces, because accelerometer measurements are biased.

The separation of the gravitational and the nongravitational forces is an important issue for gravity field determination. For gravity field determination with the CHAMP, GRACE, and GOCE missions the following cases can be distinguished (where we use the Class I and Class II observables as introduced in Sect. 2.2):

1. Use of the Class I observables (e.g., kinematic positions when determining the gravity field from GPS only, kinematic positions and $\mathrm{K}$-Band range-rates or ranges in the case of the GRACE mission)

2. Use of Class I and of Class II observables (in particular of accelerometer data). 
In the first case the influence of the non-gravitational forces is absorbed either by the parameters of physical models (for drag, direct and albedo radiation pressure, etc.), or by the parameters of the empirical forces (constant, once-, twice-, etc. per-revolution), or by stochastic parameters (pulses or piecewise constant accelerations), or by the gravity field parameters (what unfortunately never can be completely avoided), or by a combination of these quantities.

Eventually, the separation between the gravitational and non-gravitational parts of the force field has to be left to "mathematics", i.e., to the solution of the resulting combined NEQs. This separation is a delicate issue: when, e.g., setting up too many loosely constrained piecewise constant accelerations, the entire gravity signal may be absorbed, as well, by the above mentioned dynamic empirical or pseudo-stochastic parameters.

It is easy to use accelerometer data as "error free" biased and scaled accelerations in a numerical integration procedure. The bias (per component) can be simply an offset or it can be more complex by including other empirical functions (once-, etc. per-revolution, polynomial in time, etc.). When using multistep or collocation methods for numerical integration, the interval of the entire arc is subdivided into subintervals and the accelerometer time series are de facto approximated within each subinterval by polynomials of degree $q-2$, if $q$ is the order of the integration method (Beutler 2005).

\section{The celestial mechanics method}

The CMA is based on the orbit determination module of the Bernese GPS Software (Dach et al. 2007), which is used every day by the Center for Orbit Determination in Europe (CODE) Analysis Center of the IGS to determine the orbits of all GNSS satellites with openly available signals. This nucleus of the CMA already is capable of solving for pseudostochastic pulses for navigation-type satellites (Beutler et al. 1994). For gravity field determination based on the orbits of LEOs the module had to be generalized to allow for the introduction of many pulses, piecewise constant, or piecewise linear accelerations for determining the orbits of LEOs, an issue documented by Jäggi et al. (2006). The CMA was then systematically upgraded for gravity field determination using single satellites equipped with spaceborne GPS receivers and constellations equipped with GPS and ultraprecise inter-satellite measurements. Essential mathematical tools were already published in Beutler (2005), first gravity fields using CHAMP and GRACE hl-SST data were presented by Prange et al. (2009) and Jäggi et al. (2010a), respectively, a field based on 6 years of CHAMP data were subsequently generated by Prange et al. (2010), and important technical aspects related to gravity field determination using the data of spaceborne GPS receivers were discussed by Jäggi et al. (2009a). The inter-satellite capabilities of the CMA were tested for the first time to derive a gravity field based on GRACE 11-SST data gathered in 2003 (Jäggi et al. 2010a), the GRACE gravity field AIUB-GRACE02Sp based on 1 year of GRACE data (year 2007) was generated in 2008/2009 and presented by Jäggi et al. (2009b). The gravity field AIUB-GRACE02S, based on the GRACE data of the years 2006 and 2007 was presented by Jäggi et al. (2010b).

The important characteristics of the CMA are described in this section, where the discussion follows in essence the pattern of Sect. 2.

\subsection{The equations of motion and their solution}

So far, the linearized equations of motion for a particular arc were written in a generic way using the parameters $p_{i}, i=$ $1,2, \ldots, n_{\text {par }}$, see Eq. (1). In order to discuss the solution methods of the variational equations we have to be more specific. As constellations of satellites with inter-satellite measurements are an important issue, it is also required to set up simultaneous arcs for at least two satellites. This is why two satellite arcs (with identical boundaries) with indices $j=1,2$ are considered from now on. For the arc with index $j$ we distinguish

- the six parameters $p_{j 1}, p_{j 2}, \ldots, p_{j 6}$ defining the initial state vector of the arc,

- dynamic parameters, in particular

- scaling parameters $q_{i}, i=1,2, \ldots, d$ of general models relevant for all satellites and arcs,

- scaling parameters of arc-specific (usually empirical) model parameters $q_{j i}, i=1,2, \ldots, d^{\prime}$.

The gravity field parameters are typical general parameters (labelled $q_{i}$ ), constant and periodic terms in given directions are typical arc-specific parameters (labelled $q_{j i}$ ).

The initial value problems at $t_{0}$ related to the two arcs of two Earth orbiting satellites with indices $j=1,2$ may be expressed as:

$$
\begin{aligned}
\ddot{\mathbf{r}}_{j}= & -G M \frac{\mathbf{r}_{j}}{r_{j}^{3}}+\mathbf{f}\left(t, \mathbf{r}_{j}, \dot{\mathbf{r}}_{j}, q_{1}, \ldots, q_{d},\right. \\
& \left.q_{j 1}, q_{j 2}, \ldots, q_{j d^{\prime}}\right) \doteq \mathbf{f}_{j} \\
\mathbf{r}_{j 0}= & \mathbf{r}\left(t_{0} ; a_{j 0}, e_{j 0}, i_{j 0}, \Omega_{j 0}, \omega_{j 0}, u_{j 0}\right) \\
\mathbf{v}_{j 0}= & \dot{\mathbf{r}}\left(t_{0} ; a_{j 0}, e_{j 0}, i_{j 0}, \Omega_{j 0}, \omega_{j 0}, u_{j 0}\right), \quad j=1,2 .
\end{aligned}
$$

Equations (4) define the equations of motion and the initial state vectors $\mathbf{r}_{j}^{(k)}\left(t_{0}\right)=\mathbf{r}^{(k)}\left(t_{0} ; a_{j 0}, e_{j 0}, i_{j 0}, \Omega_{j 0}, \omega_{j 0}, u_{j 0}\right)$, $k=0,1, j=1,2$ at epoch $t_{0}$. The initial osculating elements define the initial state vector, where $a$ denotes the semi-major axis, $e$ the numerical eccentricity, $i$ the inclination w.r.t. the 
equatorial plane, $\Omega$ the right ascension of the ascending node, $\omega$ the argument of perigee, and $u$ the argument of latitude at time $t_{0}$.

Equations (4) refer to an inertial reference frame, the system $J 2000$ in our case. The Earth-fixed frame is the International Terrestrial Reference Frame (ITRF), underlying the generation of the CODE products (in general we use the ITRF-05, see Altamimi et al. 2007). The transformation parameters between the Earth-fixed and the inertial system are in part provided by CODE (polar motion and length of day) and in part by the IERS (see McCarthy and Petit 2003).

The equations of motion (4) implicitly contain many more dynamic parameters, which are related to models assumed as known in our context, e.g., the transformation parameters between the Earth-fixed and the inertial frames, the parameters defining the orbits and gravitational attraction of Sun, Moon and planets on the satellites, and the parameters defining the a priori known time varying part of the Earth's gravity field (due to atmosphere and oceans). Table 1 contains a list of the more important models. In most cases it is possible to select alternative models to describe one and the same physical phenomenon.

One might miss parametric models for the prominent nongravitational forces such as atmospheric drag, solar radiation pressure, albedo radiation pressure. They are not listed, because their effect should be captured by the accelerometers and/or the empirical dynamic models in Table 1. In the latter case, the impact of the non-gravitational forces is absorbed uniquely by the empirical accelerations in Table $1 \mathrm{and} /$ or by stochastic parameters.

\subsection{The GPS-derived observables}

The original observations of the CHAMP, GRACE, and GOCE missions are those acquired by the spaceborne GPS receivers, by the K-Band instrument, and by the accelerometers. GPS is the primary observation technique in the case of the CHAMP mission, K-Band plays this role for the GRACE mission, and the ensemble of three pairs of three-dimensional accelerometers, the gradiometer, is the primary instrument for the GOCE mission. The following discussion is confined to the role of the three observation types for the first two missions.

A PPP based on the ionosphere-free linear combination of phase observations of the LEO GPS receivers generates the positions $\mathbf{r}_{l}^{\prime}$ of a satellite at epochs $t_{l}, l=1,2, \ldots$, and, in principle, the full covariance matrix $\operatorname{cov}\left(\mathbf{r}_{l}^{\prime}\right)$ associated with them. The PPP procedure of the development version of the Bernese GPS Software (Dach et al. 2007) is used for all three missions. The kinematic positions are the GPS-derived observables in the CMA. The ensemble of the kinematic positions is sometimes referred to as kinematic orbit.
Table 1 Force models for satellite motion

\begin{tabular}{|c|c|}
\hline Characteristic & Comment \\
\hline \multicolumn{2}{|l|}{ Force field } \\
\hline \multicolumn{2}{|l|}{ Gravitational } \\
\hline Earth's gravity field & $\begin{array}{l}\text { Development of the Earth's static gravity } \\
\text { field into spherical harmonics. Maximum } \\
\text { degree } n \text { and order } m \text { selectable }\end{array}$ \\
\hline Solid Earth tides & $\begin{array}{l}\text { IERS2000 (McCarthy and Petit 2003), } \\
\text { elastic Earth }\end{array}$ \\
\hline Ocean tides & $\begin{array}{l}\text { Many models, e.g., FES2004 (Lyard et al. } \\
\text { 2006) }\end{array}$ \\
\hline Sun, Moon, Planets & $\begin{array}{l}\text { Point mass attractions, based on JPL } \\
\text { Development Ephemeris DE-405 (Jupiter, } \\
\text { Venus, and Mars used) }\end{array}$ \\
\hline De-aliasing & $\begin{array}{l}\text { Due to the atmosphere and the oceans' } \\
\text { response (see Flechtner 2005) }\end{array}$ \\
\hline \multicolumn{2}{|l|}{ Non-gravitational } \\
\hline Accelerometer & $\begin{array}{l}\text { Optionally, each set of tabular values of } \\
\text { the three accelerations measured onboard a } \\
\text { satellite may be used as empirically given } \\
\text { forces }\end{array}$ \\
\hline Empirical (constant) & $\begin{array}{l}\text { Constant accelerations in the } R-, S-\text {, and } \\
W \text {-directions }\end{array}$ \\
\hline Empirical (periodic) & $\begin{array}{l}\text { Accelerations of type } a_{c} \cos k u+a_{s} \sin k u \\
\text { in the } R-, S \text {-, and } W \text {-directions; } u=\text { argu- } \\
\text { ment of latitude, } k=1,2, \ldots, 5 \text { stand for } \\
\text { once- twice-, etc. per-revolution }\end{array}$ \\
\hline \multicolumn{2}{|l|}{ Transformation } \\
\hline Nutation & IAU2000 (McCarthy and Petit 2003) \\
\hline Subdaily polar motion & IERS2000 (McCarthy and Petit 2003) \\
\hline Mean pole & According to McCarthy and Petit (2003) \\
\hline Polar motion & Values of the CODE Analysis Center used \\
\hline UT & $\begin{array}{l}\text { CODE values for length of day (LoD), } \\
\text { initial value fixed on VLBI-derived series } \\
\text { (from IERS) }\end{array}$ \\
\hline
\end{tabular}

The use of the kinematic positions instead of the original GPS observations is statistically correct, if the full covariance matrix of the PPP is used. Currently, it is feasible in the CMA to make use of the full covariance matrix up to time intervals of about one revolution period for a 30 s sampling. Usually, however, the epoch-specific covariance matrices associated with the kinematic positions are used to define the epochspecific weight matrices of the kinematic positions:

$\mathbf{P}_{l} \doteq \sigma_{\mathrm{ph}}^{2}\left[\operatorname{cov}\left(\mathbf{r}_{l}^{\prime}\right)\right]^{-1}$,

where the covariance matrix refers to the a priori error $\sigma_{\mathrm{ph}}$. $\sigma_{\mathrm{ph}}$ is the RMS error a priori of the GPS phase observation of the $L 1$ carrier phase, where $\sigma_{\mathrm{ph}} \approx 0.001 \mathrm{~m}$ for the Blackjack receiver combined with a choke ring antenna (Montenbruck et al. 2006).

With definition (5) of the weight matrix, the RMS error in the parameter determination process(es) based on the GPS positions $\mathbf{r}_{j i}^{\prime}$ has the same meaning as in the preceding PPPprocedure using the original GPS observations.

Alternatively, a diagonal weight matrix in the $(R, S, W)$ system may be used. Prange et al. (2009) showed, however, 
that the choice (5) gives better results than a diagonal weight matrix.

\subsection{The filtered K-Band observations}

The biased GRACE Level 1a K-Band ranges, generated at a $10 \mathrm{~Hz}$ rate, are not publicly available. The Level 1a data are filtered. The filtering process substantially decimates the number of observation equations (by a factor of 50) without losing the accuracy inherent in the original data and without changing the information content in the bandwidth important for gravity field determination. The filter used to transform the GRACE Level 1a into the Level $1 \mathrm{~b}$ data is described by Thomas (1999). The filter is linear, i.e., the ensemble of filtered Level $1 \mathrm{~b} 5 \mathrm{~s}$ data $\rho$ is a linear combination of the ensemble of $10 \mathrm{~Hz}$ Level 1a data p. As the filter also provides the first and second time derivatives of $\rho$ we may write:

$\rho=\mathbf{D} \mathbf{p}$

$\dot{\rho}=\dot{\mathbf{D}} \mathbf{p}$

$\ddot{\rho}=\ddot{\mathbf{D}} \mathbf{p}$.

The matrices $\mathbf{D}, \dot{\mathbf{D}}$ and $\ddot{\mathbf{D}}$ are band-diagonal. Each filtered value is based on 707 consecutive unfiltered values (contained in a time window of $70.7 \mathrm{~s}$ ). As subsequent filtered values are based on heavily overlapping time windows, the correlations between the filtered values must be studied in the analysis of Level $1 \mathrm{~b}$ data.

Assuming that the Level 1a ranges are statistically independent and of the same accuracy, it is easily possible to compute the covariance matrix of the entire set of filtered values (ranges, range-rates, or range-accelerations) as a function of the covariance matrix of the errors in the unfiltered measurements:

$\operatorname{cov}(\boldsymbol{\rho})=\sigma_{k b d}^{2} \mathbf{D D}^{T}$,

where $\sigma_{k b d}$ is the RMS error of the Level 1a ranges.

The corresponding weight matrix is

$\mathbf{P}=\left(\mathbf{D D}^{T}\right)^{-1}$.

The weight matrices corresponding to range-rate and rangeaccelerations may be written as

$\mathbf{P}^{\prime}=\sigma_{k b d}^{2} \operatorname{cov}(\dot{\rho})^{-1}=\left(\dot{\mathbf{D}} \dot{\mathbf{D}}^{T}\right)^{-1}$

and

$\mathbf{P}^{\prime \prime}=\sigma_{k b d}^{2} \operatorname{cov}(\ddot{\boldsymbol{\rho}})^{-1}=\left(\ddot{\mathbf{D}} \ddot{\mathbf{D}}^{T}\right)^{-1}$.

The CMA allows it to analyze range or range-rate Level $1 \mathrm{~b}$ K-Band data. Optionally, the correlations may be taken into account when analyzing the K-Band data, however, not over the entire arc (1 day), but over user-specified time intervals of the order of $15-60 \mathrm{~min}$. It is also possible to analyze subsequent range differences or range-double-differences (without taking correlations into account). The issue is studied in detail by Beutler et al. (2010).

\subsection{Accelerometer measurements}

The correct, but unrealistic way of dealing with the accelerometer observations was outlined in Sect. 2.2. The discussion was based on the original Level 1a measurements, often assumed to be independent. The Level $1 \mathrm{~b}$ accelerations, generated at a $1 \mathrm{~s} \mathrm{spacing}$, are filtered using a similar filter (double window width, no polynomial fit, see Thomas 1999) as that used for the K-Band observations. The statistical properties of the Level $1 \mathrm{~b}$ accelerations, emerging from the filter process, are thus known and might be taken into account (for the observations of Classes I and II). The pulses or piecewise constant accelerations, the so-called pseudo-stochastic parameters (see Sect. 3.9), are an important style element of the CMA. When setting up the pulses with the spacing of the accelerometer Level 1a measurements and when using observation equations of type (3) for the accelerometer measurements, the statistical treatment of the observations would be correct. In the CMA the pseudo-stochastic parameters are currently set up with a spacing of only 5-15 min, which is of course far from the theoretically required spacing dictated by the accelerometer measurements. The discussion concerning the statistical treatment of the accelerometer measurements will be continued in Beutler et al. (2010).

\subsection{Primary equations}

When dealing with orbit or gravity field determination, each arc is represented as a linear function of the unknown parameters according to Eq. (1), implying that particular solutions of the initial value problems (4) with given a priori values for the dynamic parameters and a given initial state vector have to be generated. The computed values of the observables (the term "c" of "o-c") have to be calculated as a function of the known a priori values of the parameters with an intrinsic accuracy better than the accuracy of the observations.

The collocation method of selectable order $q=8-12$, exactly as described by Beutler (2005), was initially used in the CMA to solve the initial value problems (4). Internally, each component of the solution vector is represented piecewise (in consecutive, non-overlapping and contiguous subintervals of length $H$ covering the entire arc) by polynomials of degree $q$. The stepsize $H$ may be either defined automatically or set to a constant value. If no accelerometer-derived empirical forces are used, an algorithm with automatic stepsize control can be used. 
Fig. 2 Accumulated rounding errors in $R$-, $S$ - and $W$-directions; left conventional collocation method, right modified collocation method (note the scale differences in the figures)
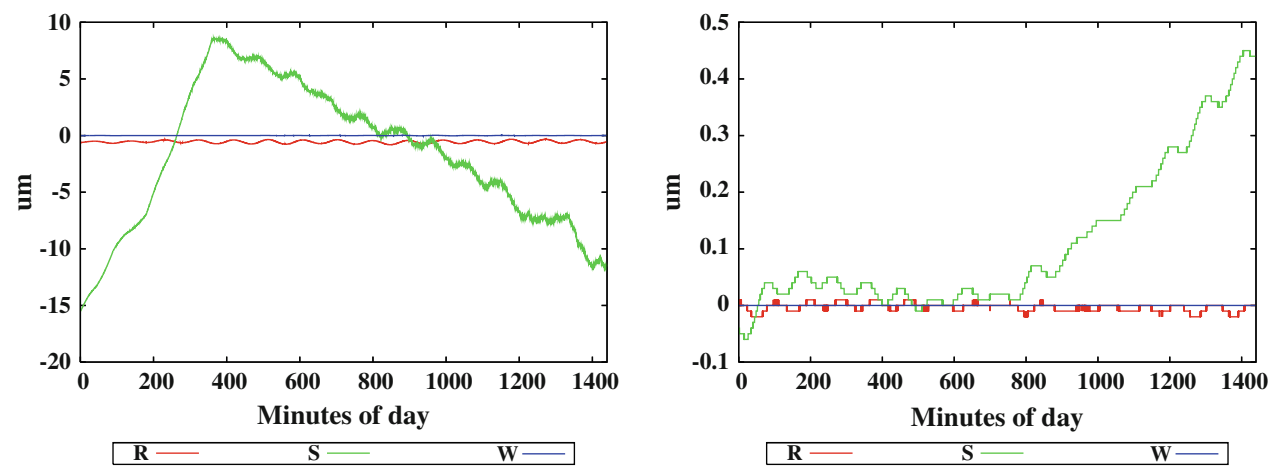

It is not trivial to generate solutions of the initial value problems (4), from which inter-satellite distances with an accuracy of about $1 \mu \mathrm{m}$ may be derived for arcs as long as 1 day. In order to check the performance of our integrator, the following experiment was performed: an "errorfree" series of satellite positions with a spacing of $10 \mathrm{~s}$ was produced using the collocation method of order $q=8$ with automatic stepsize control. The Earth's gravity field EigenCG03C (Förste et al. 2008) was cut off after the degree $n_{\max }=150$. The third-body perturbations, the fixed-body and the ocean tides were taken into account. Air drag and radiation pressure were ignored. These positions were then used (with the same force field) with a collocation procedure of order $q=9$ and a slightly different setting for the automatic stepsize control in an iterative orbit improvement process (to avoid the generation of identical rounding errors in the simulation and estimation procedures), where only the six initial osculating elements were determined. One would expect the residuals, i.e., the differences between the simulated and estimated positions, to be below the $1 \mu \mathrm{m}$ level. The average stepsize $\bar{H}$ was 11.2 and 16.2 s for the simulation and the orbit determination run, respectively. Figure 2 (left) shows the errors in the $R$-, $S-, W$-components over the entire day using the collocation method provided by Beutler (2005). The resulting residuals are clearly above the required accuracy level. Differences of up to about $15 \mu \mathrm{m}$ show up between the simulated and estimated $S$-component of the satellite positions. The same figure also proves that the conventional procedure may be used without concerns for gravity field determination based "only" on GPS-derived positions: the resulting inconsistencies are clearly below the accuracy of the kinematic satellite positions.

In order to guarantee accuracies $<1 \mu \mathrm{m}$ for inter-satellite distances, the collocation procedure in Beutler (2005) was modified to represent the initial state vectors associated with the subintervals with better than double precision (corresponding to 64 bits assigned to a floating point number). This can be achieved easily, as the individual integration step at epoch $t_{i}$ of our numerical integration procedure solving the system of type (4) for one of the satellites can be brought into the form
$\mathbf{r}\left(t_{i+1}\right)=\mathbf{r}\left(t_{i}\right)+\Delta \mathbf{r}_{i, i+1}$

$\dot{\mathbf{r}}\left(t_{i+1}\right)=\dot{\mathbf{r}}\left(t_{i}\right)+\Delta \dot{\mathbf{r}}_{i, i+1}$

and because the absolute values of the increments are much smaller than those of the state vector at $t_{i}$. It is thus sufficient in a first order to store only the initial state vector $\mathbf{r}\left(t_{i}\right), \dot{\mathbf{r}}\left(t_{i}\right)$ with higher than double precision and to calculate only the sums in Eq. (11) in extended precision. The modified procedure reduces the rounding error by several orders of magnitude.

The sketched modified procedure has roughly the same characteristics concerning computational efficiency and storage requirements as the conventional method, but reduces the accumulated rounding errors below the required error level. Figure 2 (right) shows the success of the procedure.

The example indicates that FORTRAN "double precision" (with 64 bits per double precision number) reaches its limits for our demanding application. A general change to "extended precision" (with 128 bits per extended precision number) is currently not considered. With increasing computing power, better processors, and improved FORTRAN compilers, this rigorous solution might become feasible in future for the purpose of numerical integration, which might be important for the analysis of future gravity missions based on even more accurate inter-satellite data such as interferometric laser measurements.

Equation (1) is used for orbit and gravity field determination. The equation was obtained by linearizing the original equations of motion (4). It is important to keep the linearization errors in the satellite orbits small. For gravity field determination using kinematic positions and inter-satellite measurements the a priori orbits are established by the following four-step procedure (described in more detail by Jäggi et al. 2010b):

1. Approximate orbit parameters in Eqs. (4) are obtained, separately for each arc and satellite, with an iterative orbit determination procedure using an a priori gravity field and using only the kinematically established satellite positions as pseudo-observations (together with the associated weight matrices) to determine the six initial 
osculating elements per arc and the other satellite- and arc-specific parameters (pulses or piecewise constant accelerations) as unknowns, with a spacing foreseen for the "final" solution. The corresponding NEQs are saved.

2. The orbits resulting from step 1 are used to set up the normal equations using the K-Band observations (with the same parametrization). The resulting NEQs contain the arc-specific parameters for both satellites.

3. The two GPS NEQs and the K-Band NEQ are now combined, using a realistic weight ratio of the two observable types to derive the a priori orbits for the satellites of the constellation. The resulting orbits represent the kinematic positions with an accuracy of few $\mathrm{cm}$, the K-Band observations with an accuracy of few $\mu \mathrm{m}$ for range or about $0.1-0.2 \mu \mathrm{m} / \mathrm{s}$ for range-rate (if a good a priori field is used).

4. The orbits resulting from step 3 (based on GPS and $\mathrm{K}$-Band) are used as a priori orbits for gravity field determination. In this "final" step the parameter list has to be enlarged to contain the gravity field parameters, as well. Note that three NEQs are set up, namely two GPSspecific related to the kinematic positions of the two satellites and one related to the K-Band observations (if the parameter transformation of Sect. 3.14 is applied, the two GPS NEQs are merged into one GPS NEQ before combining the resulting GPS NEQ with the K-Band NEQ).

The quality of the a priori gravity field is not critical in this procedure-even the EGM96 (Lemoine et al. 1997) is sufficient. The truth of this statement was demonstrated by Jäggi et al. (2010a). For gravity field determination based only on the GPS-derived kinematic positions the entire procedure is reduced to the first step-with the exception that the gravity field parameters have to be set up, as well, in the last step of the iterative orbit improvement and stored in the corresponding NEQ.

\subsection{Arcs, arc length and pulses}

One set of initial osculating elements might in principle be declared valid over very long time intervals (e.g., over years). The example of the preceding section showed, however, that orbital arcs with a length of 1 day and an accuracy $<1 \mu \mathrm{m}$ per coordinate are already difficult to generate with pure dynamic methods. As the data of gravity missions are usually made available in 1-day batches, arc lengths of 1 day are convenient. One day arcs are therefore used as the basic arc length in the CMA.

By allowing for instantaneous velocity changes (pulses) $\Delta v_{R l}, \Delta v_{S l}$, and $\Delta v_{W l}$, in the pre-defined directions $\mathbf{e}_{R}$, $\mathbf{e}_{S}, \mathbf{e}_{W}$ and at user-defined epochs $t_{p l}, l=1,2, \ldots, n_{s}$ with a spacing of $\Delta t_{p}$ min, it is easily possible to generate many different contiguous short-arc solutions with lengths of $\Delta t_{p}$ min or multiples thereof. In the CMA we usually select $\Delta t_{p}$ between 5 and $30 \mathrm{~min}$. The short arcs generated by the CMA differ from "normal" short arcs, which are described by a full set of six initial or boundary values. Normal short arcs are not contiguous.

The actual selection of the short-arc length takes place when stacking the sets of the daily NEQs. The additional storage requirements for one daily NEQ with pulses are modest: for a $\Delta t_{p}=15$ min spacing between subsequent pulses $3 \times 95 \approx 300$ additional parameters have to be added to the NEQs without pulses. This additional burden does almost not matter for gravity field determination, where the parameters are typically counted in tens of thousands.

By setting up in addition to the pulses offsets in three orthogonal directions at the boundary epochs it would be easily possible to modify the CMA procedure to make it fully equivalent to a conventional short-arc method. As a similar effect in the data fit may be achieved by reducing the time interval $\Delta t_{p}$ between pulses, as well, this generalization was not implemented into the CMA.

\subsection{Variational equations}

With the introduction of pulses at $n_{s}$ epochs separated by $\Delta t_{p}$ the list of parameters defining the arc with index $j$ reads as:

$$
\begin{aligned}
& \left\{p_{j 1}, p_{j 2}, \ldots, p_{j, 6+d+d^{\prime}+3 n_{s}}\right\} \\
& \quad \doteq\left\{a_{j}, e_{j}, i_{j}, \Omega_{j}, \omega_{j}, u_{j 0}, q_{1}, \ldots, q_{d}, q_{j 1}, \ldots, q_{j d^{\prime}},\right. \\
& \quad \Delta v_{j R_{1}}, \Delta v_{j R_{2}}, \ldots, \Delta v_{j R_{n_{s}}}, \\
& \quad \Delta v_{j S_{1}}, \Delta v_{j S_{2}}, \ldots, \Delta v_{j S_{n_{s}}}, \\
& \left.\quad \Delta v_{j W_{1}}, \Delta v_{j W_{2}}, \ldots, \Delta v_{j W_{n_{s}}}\right\} .
\end{aligned}
$$

Each arc is thus defined by six initial osculating elements, $3 n_{s}$ pulses, $d^{\prime}$ satellite- and arc-specific, and $d$ general dynamic parameters. Each orbit may thus be written in linearized form as stated in Eq. (1). Introducing for abbreviation the symbol

$\mathbf{z}_{j k}(t) \doteq \frac{\partial \mathbf{r}_{0 j}(t)}{\partial p_{j k}}$,

where $\mathbf{r}_{0 j}(t)$ define the a priori orbits based on the sets $p_{j 01}, p_{j 02}, \ldots, p_{j 0,6+d+d^{\prime}+3 n_{s}}$ of known approximate values of the parameters (12) (the understanding of the functions $\mathbf{r}_{0 j}(t)$ and of the partial derivatives of them is the same as that related to the corresponding symbols in Eq. (1)). Taking the derivative of Eqs. (4) w.r.t. parameter $p_{j k}$ one obtains the so-called variational equations for this parameter together with the corresponding initial values:

$\ddot{\mathbf{z}}_{j k}=\mathbf{A}_{j 0} \cdot \mathbf{z}_{j k}+\mathbf{A}_{j 1} \cdot \dot{\mathbf{z}}_{j k}+\frac{\partial \mathbf{f}_{j_{0}}}{\partial p_{j k}}$
$\mathbf{z}_{j k}\left(t_{0}\right)=\frac{\partial}{\partial p_{j k}}\left\{\mathbf{r}\left(p_{j 01}, p_{j 02}, \ldots, p_{j 0,6+d+d^{\prime}+3 n_{s}}\right)\right\}$ 


$$
\begin{aligned}
& \doteq \frac{\partial \mathbf{r}_{j_{0}}}{\partial p_{j k}} \\
\dot{\mathbf{z}}_{j k}\left(t_{0}\right) & =\frac{\partial}{\partial p_{j k}}\left\{\dot{\mathbf{r}}\left(p_{j 01}, p_{j 02}, \ldots, p_{\left.\left.j 0,6+d+d^{\prime}+3 n_{s}\right)\right\}}\right)\right. \\
& \doteq \frac{\partial \dot{\mathbf{r}}_{j_{0}}}{\partial p_{j k}}
\end{aligned}
$$

where the $3 \times 3$ matrices $\mathbf{A}_{j 0}$ and $\mathbf{A}_{j 1}$ are defined by

$A_{j 0[l m]}=\frac{\partial f_{j_{0_{l}}}}{\partial r_{j_{0_{m}}}}, \quad A_{j 1[l m]}=\frac{\partial f_{j_{0_{l}}}}{\partial \dot{r}_{j_{0_{m}}}}$,

and where $f_{j_{0}}$ denotes the component $l$ of the total acceleration $\mathbf{f}_{j_{0}}$ in Eqs. (4); the subscript " 0 " indicates that the force refers to the known a priori orbit. The initial values $\mathbf{z}_{j k}\left(t_{0}\right)$ and $\dot{\mathbf{z}}_{j k}\left(t_{0}\right)$ are zero for all dynamic parameters, whereas the explicit derivative of the force vector $\mathbf{f}_{j_{0}}$ w.r.t. parameter $p_{j k}$ is zero for the initial osculating elements and for the pulses. The matrices $\mathbf{A}_{j 0}$ and $\mathbf{A}_{j 1}$ are the same for all parameters, i.e., all variational equations are based on the same homogeneous system. In the absence of velocity-dependent forces we have $\mathbf{A}_{j 1}=\mathbf{0}$. Note that $\mathbf{A}_{j 1}(t) \neq \mathbf{0}$, as soon as empirical forces in directions $S$ or $W$ are set up, because the velocity vector is required to calculate the unit vectors in these directions.

The differential equation system in Eqs. (14) is linear, homogeneous and of second order with initial values $\mathbf{z}_{j k}$ $\left(t_{0}\right) \neq \mathbf{0}$ and $\dot{\mathbf{z}}_{j k}\left(t_{0}\right) \neq \mathbf{0}$, if the parameter $p_{j k}$ is either one of the initial osculating elements or one of the pulses, whereas the variational equations in Eqs. (14) are linear, but inhomogeneous for $p_{j k} \in\left\{q_{1}, \ldots, q_{d}, q_{j_{1}}, q_{j_{2}}, \ldots, q_{j_{d}^{\prime}}\right\}$, but then they have zero initial values, namely $\mathbf{z}_{j k}\left(t_{0}\right)=\dot{\mathbf{z}}_{j k}\left(t_{0}\right)=\mathbf{0}$.

According to the theory of linear differential equation systems the general solution of a homogeneous linear differential equation system of order $N$ and dimension $D$ is given by $N D$ linearly independent particular solutions. As $N=2$ and $D=3$ in the case of orbit or gravity field determination, the six solutions $\mathbf{z}_{j m}(t), m=1,2, \ldots, 6$, associated with the initial osculating elements (for each arc and satellite), may be selected as the elements of the complete system of solutions of the homogeneous part of Eqs. (14).

In the CMA the six solutions of Eqs. (14) associated with the initial osculating elements are generated by simultaneous numerical integration with the primary system (4). No use is made of the linearity of the system (14) at this stage. The partial derivatives $\mathbf{z}_{j k}(t)$ for all other parameters $p_{j k}, k>6$ are represented as linear combinations of the partial derivatives w.r.t. the six initial osculating elements:

$$
\begin{aligned}
& \mathbf{z}_{j k}(t)=\sum_{m=1}^{6} \alpha_{k j m} \mathbf{z}_{j m}(t) \\
& \dot{\mathbf{z}}_{j k}(t)=\sum_{m=1}^{6} \alpha_{k j m} \dot{\mathbf{z}}_{j m}(t),
\end{aligned}
$$

where the coefficients are time-independent, if the parameter $p_{j k}$ corresponds to a pulse, they are functions of time $t$, if $p_{j k}$ is one of the dynamic parameters. In the former case the time-independent parameters result as the solution of a linear system of equations, in the latter case they emerge as integrals (see Beutler 2005, Vol. 1, Chapter 5).

The solution of the primary equations (4) and of the six variational equations associated with the six initial osculating elements on one hand is completely separated from the solution of the variational equations for all other parameters on the other hand. The computational burden for the solution of the variational equation w.r.t. pulses is negligible in the CMA, as the corresponding partial derivatives (16) are linear combinations of the partial derivatives w.r.t. the initial osculating elements with time-independent coefficients.

The six coefficients of the linear combination (16) have to be calculated as integrals in the case of dynamical parameters. Instead of numerically solving a differential equation system of order 2 and dimension 3 one thus has to solve six definite integrals by numerical quadrature. As there are much more efficient methods available for numerical quadrature than for the numerical solution of ordinary differential equation systems, namely the Gaussian quadrature procedures, which are consistently used in the CMA, the computational burden is substantially reduced, as well, for the solution of the variational equations for the dynamic parameters. The efficiency gain is (a) due to the elimination of all iterative procedures occurring in the solvers of ordinary differential equations (each integrated value is a linear combination of a small number of values of the integrand) and (b) due to the fact that the Gaussian quadrature formulas approximate definite integrals based on $q$ values of the integrand by a polynomial (Taylor series expansion) of degree $2 q-1$ and not of $q-1$, as it would be the case for differential equations. This behavior essentially breaks error propagation.

The reduction of CPU requirements is, as a matter of fact, so significant that the coefficients $\alpha_{k j m}(t)$ are not stored and then interpolated to particular values, but actually re-calculated as integrals whenever needed.

The solution of the primary equations, the complete system of the six solutions of the homogeneous part of Eqs. (14), and the coefficients $\alpha_{k j m}$ for all pulses (or accelerations) are, however, stored together with the pulse epochs in a so-called standard orbit file, allowing the retrieval of the position vector and its time derivatives for each time argument $t$ in the arc to the accuracy needed. The information in the standard orbit file allows it therefore, as well, to retrieve the partial derivatives w.r.t. the initial osculating elements and w.r.t. all pulses. The bureaucratic burden of the procedure and the storage requirements are considerably reduced by making consequent use of the representation (16) for the partial derivatives w.r.t. the dynamic parameters. For more information we refer to Beutler (2005, Vol I, Chapters 5 and 7). 


\subsection{Pulses versus piecewise-constant accelerations}

The CMA allows it to set up piecewise constant accelerations in the $R$-, $S$-, and $W$-directions in the intervals $\left[t_{p l}, t_{p l}+\Delta t_{p}\right]$ instead of setting up pulses at the epochs $t_{p l}$. The partial derivatives w.r.t. the piecewise-constant accelerations may be computed very efficiently, as well. The partial derivatives with respect to all piecewise-constant accelerations in a particular direction may be written in the form (16), where the coefficients may be derived according to Jäggi et al. (2006) from the coefficients of the partial derivative of the orbit w.r.t. a constant acceleration in the same direction acting over the entire arc. Jäggi et al. (2006) state that piecewise-constant accelerations are preferable for orbit determination. For gravity field determination different recommendations are made (see Beutler et al. 2010).

3.9 Constraining the pulses or the piece-wise constant accelerations

The introduction of pulses or piece-wise constant accelerations is motivated by exactly the same reasons as the use of short-arcs, namely by the imperfectly known force field (with or without using accelerometer data). Compared to the classical short-arc method one is, however, in a much better position to make use of the known statistical properties of the non-modelled parts of the force field by constraining the pulses or accelerations. Theoretically one might achieve similar effects by constraining the offsets and the pulses in conventional short-arc methods, but one then would have to refer all short-arcs to one and the same a priori "longarc" orbit; this requirement would remove the simpleness and attractiveness of the conventional short-arc method to a great extent.

A pulse $\Delta v_{l}$ in a particular direction $\mathbf{e}\left(t_{l}\right)$ at time $t_{l}$ is to the first order in $\Delta t_{p}$ equivalent to an acceleration $\mathbf{a}_{l}(t)$ of constant size $\Delta a_{l}$ acting in the direction $\mathbf{e}(t)$, i.e., $\mathbf{a}_{l}(t) \doteq$ $\Delta a_{l} \mathbf{e}(t) \approx \Delta a_{l} \mathbf{e}\left(t_{l}\right)$, and in the time interval $\left[t_{l}, t_{l}+\Delta t_{p}\right]$, $\Delta t_{p}=\left|\left[t_{l}, t_{l+1}\right]\right|$. The pulse and the acceleration are thus approximately related by

$\Delta v_{l} \approx \Delta a_{l} \Delta t_{p}$.

Equation (17) should be used in particular when accounting for the impact of the stochastic part of the accelerometer data in Eq. (3) using constrained pulses.

Currently the pulses or accelerations are set up in the CMA typically with a spacing of $\Delta t_{p}=5-30 \mathrm{~min}$. In order to come up with meaningful constraints for these parameters from the point of view of theory we have to estimate their size as a function of the accelerometer Level 1a accuracy of about $1 \times 10^{-10} \mathrm{~m} / \mathrm{s}^{2}$ in the accelerations (for the more accurate axes). Assuming that the accelerometer accuracy is the only error source, this value can be transformed into an "elemen- tary" pulse accuracy of the duration of $\Delta t_{p l k, L 1}=0.1 \mathrm{~s}$ of $1 \times 10^{-11} \mathrm{~m} / \mathrm{s}$ at epochs $t_{p l k}$ regularly spaced in the interval $\left[t_{l}, t_{l}+\Delta t_{p}\right]$, where $\Delta t_{p, L 1}=0.1 \mathrm{~s}$ is the spacing of "independent" Level 1a accelerometer measurements. Assuming these elementary pulses to be independent we can assess the RMS error of the CMA pulse $\Delta v_{l}\left(\Delta t_{p}\right)$ set up every $\Delta t_{p}$ seconds approximately as

$\sigma_{v_{l}}^{2}\left(\Delta t_{p}\right)=\sum_{k=1}^{m} \sigma_{p l k, L 1}^{2}=m \cdot \sigma_{p, L 1}^{2}$,

where $m=\Delta t_{p} / \Delta t_{p, L 1}$. For pulses set up at 15-min intervals we thus have

$\sigma_{v_{l}}\left(\Delta t_{p}\right)=\sqrt{m} \cdot \sigma_{p, L 1} \approx 1 \times 10^{-9} \mathrm{~m} / \mathrm{s}$.

The corresponding value for piecewise constant accelerations can be calculated from Eq. (19) using Eq. (17).

When accelerometer data are ignored or not available and when the non-gravitational forces are accounted for only by a modest empirical model with few estimated parameters, e.g., constant and once-per-revolution terms in the three directions $R, S$, and $W$, one has to expect effects due to the residual part of the non-gravitational forces of the order of a few $10^{-9} \mathrm{~m} / \mathrm{s}^{2}$ in the accelerations (about 10-100 times smaller in the differences of the accelerations between GRACE-A and -B). As this problem can hardly be treated by statistical considerations, one should either use unconstrained solutions or find the appropriate weighting by numerical experiments. The impact of setting up constrained piecewise constant accelerations or pulses will be further discussed by Beutler et al. (2010).

\subsection{Parameter estimation: general aspects}

Parameter estimation is based on the classical least-squares method and the satellite orbits solve ordinary differential equations in the CMA. Pulses or piecewise constant accelerations with a high time resolution give the orbits a flexibility close to that offered by a filter approach based on stochastic differential equation systems. The relationship between least-squares method based on pulses and accelerations on one hand and the filter approaches on the other hand was treated by Beutler et al. (2006).

The NEQ contributions are set up separately for the GPS and the K-Band observables. In principle the contributions of the accelerometers are also set up separately, but the resulting NEQs are not made available as separate entities. The GPS contributions are generated separately for all satellites and arcs involved. It is thus possible to derive gravity fields using only GPS (even for individual satellites) and to find the correct weight ratio of the K-Band and GPS-contributions (Beutler et al. 2010). 


\subsection{Parameter estimation: the GPS contribution}

For the solution of the variational equations the distinction was made between parameters defining the initial state vector, the force field, and the stochastic parameters. In this section we only make the distinction between arc-and satellite-specific parameters on one hand and general parameters occurring in different NEQs. The parameters of the former kind define the underlying orbit determination problem, the parameters of the latter kind, e.g., the gravity field.

The linearized observation equation for the position of satellite $j$ at time $t_{l}$ may thus be written as:

$\mathbf{A}_{o, j l} \mathbf{o}_{j}+\mathbf{A}_{q, j l} \mathbf{q}-\Delta \mathbf{r}_{j l}=\mathbf{v}_{j l}$,

where $\mathbf{o}_{j}$ is the array containing all parameters defining the initial conditions, all satellite- and arc-specific dynamic parameters, and all stochastic pulses, $\mathbf{q}$ is the array with the common dynamic parameters, $\Delta \mathbf{r}_{j l} \doteq \mathbf{r}_{j l}^{\prime}-\mathbf{r}_{0 j}\left(t_{l}\right)$ contains the terms "observed - computed" for the satellite and time considered, and $\mathbf{v}_{j l}$ is the array with the residuals. $\mathbf{A}_{o, j l}$ and $\mathbf{A}_{q, j l}$ are the first design matrices corresponding to the parameter arrays $\mathbf{o}_{j}$ and $\mathbf{q}$. The first design matrices $\mathbf{A}_{\ldots}$.. contain three lines corresponding to the three Cartesian coordinates. The number of columns corresponds to the number of parameters of the particular type. Each column of any of the matrices $\mathbf{A}_{\text {... }}$ may be written as

$\mathbf{A}_{\ldots, k}=\frac{\partial \mathbf{r}_{0 j}\left(t_{l}\right)}{\partial p_{j k}}$,

where $p_{j k}$ is the parameter pertaining to column $k$ (the understanding of the functions $\mathbf{r}_{0 j}(t)$ and of their partial derivatives is the same as that related to the corresponding symbols in Eq. (1)).

Assuming an epoch specific weight matrix according to Eq. (5) the NEQ contribution arising from the kinematic position of satellite $j$ at time $t_{l}$ is:

$$
\begin{aligned}
& \left(\begin{array}{lll}
\mathbf{A}_{o, j l}^{T} \mathbf{P}_{j l} \mathbf{A}_{o, j l} & , & \mathbf{A}_{o, j l}^{T} \mathbf{P}_{j l} \mathbf{A}_{q, j l} \\
\mathbf{A}_{q, j l}^{T} \mathbf{P}_{j l} \mathbf{A}_{o, j l} & , & \mathbf{A}_{q, j l}^{T} \mathbf{P}_{j l} \mathbf{A}_{q, j l}
\end{array}\right)\left(\begin{array}{c}
\mathbf{o}_{j} \\
\mathbf{q}
\end{array}\right) \\
& =\left(\begin{array}{cc}
\mathbf{A}_{o, j l}^{T} \mathbf{P}_{j l} \Delta \mathbf{r}_{j l} \\
\mathbf{A}_{q, j l}^{T} \mathbf{P}_{j l} \Delta \mathbf{r}_{j l}
\end{array}\right) .
\end{aligned}
$$

The total NEQ contribution for one satellite and for one arc is simply obtained by summing up all contributions (22) for $l=1,2, \ldots$. In an attempt to further simplify matters the NEQs for the two satellites of the constellation are written as:

$$
\begin{aligned}
& \left(\begin{array}{ll}
\mathbf{N}_{11} & \mathbf{N}_{1 q} \\
\mathbf{N}_{1 q}^{T} & \mathbf{N}_{q q}
\end{array}\right)\left(\begin{array}{c}
\mathbf{o}_{1} \\
\mathbf{q}
\end{array}\right)=\left(\begin{array}{l}
\mathbf{b}_{1} \\
\mathbf{b}_{q}
\end{array}\right) \text { and } \\
& \left(\begin{array}{ll}
\mathbf{M}_{22} & \mathbf{M}_{2 q} \\
\mathbf{M}_{2 q}^{T} & \mathbf{M}_{q q}
\end{array}\right)\left(\begin{array}{c}
\mathbf{o}_{2} \\
\mathbf{q}
\end{array}\right)=\left(\begin{array}{l}
\mathbf{c}_{2} \\
\mathbf{c}_{q}
\end{array}\right) .
\end{aligned}
$$

Gravity field determination based only on the positions of the satellites is completed by stacking the arc-specific contributions (23) — after having pre-eliminated the arc-specific parameters. The pre-elimination results in the following reduced NEQ for the first satellite:

$\left[\mathbf{N}_{q q}-\mathbf{N}_{1 q}^{T} \mathbf{N}_{11}^{-1} \mathbf{N}_{1 q}\right] \mathbf{q}=\mathbf{b}_{q}-\mathbf{N}_{1 q}^{T} \mathbf{N}_{11}^{-1} \mathbf{b}_{1}$

GPS-specific weekly, monthly, annual, etc., solutions are obtained by stacking the reduced daily NEQs (24). From the formal point of view it does not matter whether only one or several different satellites are involved. One expects of course better results if satellites with different orbit characteristics contribute to a particular solution. This statement is based on the experiences of the pre-GPS era, when the global gravity field had to be reconstructed mainly from the SLR and astrometric tracking techniques (see Biancale et al. 2000).

\subsection{Parameter estimation: the K-Band contribution}

As in the case of the GPS observation equations we distinguish (a) satellite- and arc-specific and (b) common parameters. A "new" parameter type, namely the K-Band bias parameters, has to be considered when analyzing ranges, because the K-Band range measurements are biased, (rangerates are not biased). Designating the new array of common parameters containing all parameters related to the force field and the range biases by $\tilde{\mathbf{q}}$, the observation equation for the inter-satellite distance $d\left(t_{l}\right)$ at time $t_{l}$ may then be written as

$\tilde{\mathbf{A}}_{o, 1 l} \mathbf{0}_{1}+\tilde{\mathbf{A}}_{o, 2 l} \mathbf{o}_{2}+\tilde{\mathbf{A}}_{q l} \tilde{\mathbf{q}}-\Delta d_{l}=v_{l}$,

where $\tilde{\mathbf{A}}_{o, \ldots}$ are the first design matrices of the observed distance corresponding to the satellite- and arc-specific parameters for the two satellites and where $\tilde{\mathbf{A}}_{q l}$ is the first design matrix corresponding to the common parameters. $\Delta d_{l}=\rho_{l}-$ $d\left(t_{l}\right)$ is the term "observed distance - a priori value for the distance" at time $t_{l}$, and

$d\left(t_{l}\right)=\left|\mathbf{r}_{02}\left(t_{l}\right)-\mathbf{r}_{01}\left(t_{l}\right)\right|$

is the distance as derived from the particular solutions of the equations of motion at time $t_{l}$ for the two satellites considered.

For a particular satellite-specific parameter we have

$$
\begin{aligned}
& \tilde{A}_{o, 1 l ; k}=-\frac{\mathbf{r}_{02}\left(t_{l}\right)-\mathbf{r}_{01}\left(t_{l}\right)}{d\left(t_{l}\right)} \cdot \frac{\partial \mathbf{r}_{01}\left(t_{l}\right)}{\partial o_{1, k}} \text { and } \\
& \tilde{A}_{o, 2 l ; k}=+\frac{\mathbf{r}_{02}\left(t_{l}\right)-\mathbf{r}_{01}\left(t_{l}\right)}{d\left(t_{l}\right)} \cdot \frac{\partial \mathbf{r}_{02}\left(t_{l}\right)}{\partial o_{2, k}}
\end{aligned}
$$

and for a common dynamic parameters $q_{k}$

$$
\tilde{A}_{q l, k}=\frac{\mathbf{r}_{02}\left(t_{l}\right)-\mathbf{r}_{01}\left(t_{l}\right)}{d\left(t_{l}\right)} \cdot\left[\frac{\partial \mathbf{r}_{02}\left(t_{l}\right)}{\partial q_{k}}-\frac{\partial \mathbf{r}_{01}\left(t_{l}\right)}{\partial q_{k}}\right] .
$$


If the element No. $k$ of $\tilde{\mathbf{A}}_{q l}$ corresponds to a bias parameter (active at $t_{l}$ ) we have

$\tilde{A}_{q l, k}=1$.

In the most general case a fully populated weight matrix $\mathbf{P}$ has to be taken into account when creating the NEQ. It makes therefore sense to write all K-Band specific observation equations of one arc (actually: all K-Band observations within an interval in which the correlations are modelled correctly) in matrix form

$\tilde{\mathbf{A}}_{o, 1} \mathbf{o}_{1}+\tilde{\mathbf{A}}_{o, 2} \mathbf{o}_{2}+\tilde{\mathbf{A}}_{q} \tilde{\mathbf{q}}-\Delta \mathbf{d}=\mathbf{v}$,

where line $l$ of matrix $\tilde{\mathbf{A}}_{o, j}, j=1,2$ is the matrix $\tilde{\mathbf{A}}_{o, j l}$, etc. Assuming that $\mathbf{P}$ is the weight matrix of the entire set of measurements, the resulting NEQ assumes the form

$$
\begin{aligned}
& \left(\begin{array}{ccc}
\tilde{\mathbf{A}}_{o, 1}^{T} \mathbf{P} \tilde{\mathbf{A}}_{o, 1}, & \tilde{\mathbf{A}}_{o, 1}^{T} \mathbf{P} \tilde{\mathbf{A}}_{o, 2}, & \tilde{\mathbf{A}}_{o, 1}^{T} \mathbf{P} \tilde{\mathbf{A}}_{q} \\
\tilde{\mathbf{A}}_{o, 2}^{T} \mathbf{P} \tilde{\mathbf{A}}_{o, 1}, & \tilde{\mathbf{A}}_{o, 2}^{T} \mathbf{P} \tilde{\mathbf{A}}_{o, 2}, & \tilde{\mathbf{A}}_{o, 2}^{T} \mathbf{P} \tilde{\mathbf{A}}_{q} \\
\tilde{\mathbf{A}}_{q}^{T} \mathbf{P} \tilde{\mathbf{A}}_{o, 1}, & \tilde{\mathbf{A}}_{q}^{T} \mathbf{P} \tilde{\mathbf{A}}_{o, 2}, & \tilde{\mathbf{A}}_{q}^{T} \mathbf{P} \tilde{\mathbf{A}}_{q}
\end{array}\right)\left(\begin{array}{c}
\mathbf{o}_{1} \\
\mathbf{o}_{2} \\
\tilde{\mathbf{q}}
\end{array}\right) \\
& =\left(\begin{array}{c}
\tilde{\mathbf{A}}_{o, 1}^{T} \mathbf{P} \Delta \mathbf{d} \\
\tilde{\mathbf{A}}_{o, 2}^{T} \mathbf{P} \Delta \mathbf{d} \\
\tilde{\mathbf{A}}_{q}^{T} \mathbf{P} \Delta \mathbf{d}
\end{array}\right) \text {. }
\end{aligned}
$$

\subsection{Parameter estimation: combining the GPS} and K-Band contributions

The NEQ (31) containing the K-Band contribution must now be combined with the GPS-systems (23). The only open issue is the determination of the correct ratio of the RMS errors $\sigma_{\mathrm{ph}} / \sigma_{k b d}$ of the two measurement techniques, which has to be taken into account when stacking the K-Band and the GPS NEQ contributions.

It is important to note that this ratio is of the order of $\sigma_{\mathrm{ph}} / \sigma_{k b d} \approx 1,000$ when using K-Band ranges, it is of the order of $\sigma_{\mathrm{ph}} / \sigma_{k b d} \approx 10,000 \mathrm{~s}$ when using K-Band rangerates. Such ratios may give rise to numerical problems when combining the GPS- and K-Band-specific NEQs, because both contributions have to be referred to one and the same mean error a priori (in the case of the CMA either to $\sigma_{\mathrm{ph}}$ or to $\left.\sigma_{k b d}\right)$. A scaling of the K-Band system with a factor of $\sigma_{\mathrm{ph}}^{2} / \sigma_{k b d}^{2}$ (adopting $\sigma_{\mathrm{ph}}$ as weight unit) will lead to the loss of many significant digits of the GPS contribution. This particular numerical problem is significantly reduced by applying the transformation proposed in the next paragraph.

\subsection{Parameter estimation: transformation of orbital parameters for constellations}

When dealing with constellations of satellites separated by a few hundred km only it is not advisable to use the NEQs and the stacking procedure given in the previous section. In order to illustrate the problems involved, we analyze the structure of the observation equation (25) related to the inter-satellite distance $d\left(t_{l}\right)$ of two satellites.

If the two satellites are close in space (in the case of the GRACE mission the distance $d(t)$ varies between about 170 and $270 \mathrm{~km}$ ) we may conclude from Eqs. (27):

$\tilde{\mathbf{A}}_{o, 2 l} \approx-\tilde{\mathbf{A}}_{o, 1 l}$.

Whether or not a particular element $\tilde{A}_{q l, k}$ of the line matrix $\tilde{\mathbf{A}}_{q l}$ is small depends on the wavelength of the particular term of the gravity field. If the element $A_{q l, k}$ corresponds to a term of low degree, it is actually small in absolute value, which is why the K-Band measurements are not well suited to determine the low degree terms. If the wavelength is, however, of the order of the distance $d \approx 200 \mathrm{~km}$ between the satellites, this will in general not be the case.

Equation (32) shows that the satellite positions themselves are not well established with equations of type (25). This is why the following parameter transformation is performed:

$\mathbf{p}_{1} \doteq \frac{1}{2}\left[\mathbf{o}_{1}+\mathbf{o}_{2}\right], \quad \mathbf{o}_{1}=\mathbf{p}_{1}+\mathbf{p}_{2}$

$\mathbf{p}_{2} \doteq \frac{1}{2}\left[\mathbf{o}_{1}-\mathbf{o}_{2}\right], \quad \mathbf{o}_{2}=\mathbf{p}_{1}-\mathbf{p}_{2}$.

Using transformation (33) the observation equations (25), $l=1,2, \ldots$ may be modified as follows:

$$
\left[\mathbf{A}_{1}+\mathbf{A}_{2}\right] \mathbf{p}_{1}+\left[\mathbf{A}_{1}-\mathbf{A}_{2}\right] \mathbf{p}_{2}+\mathbf{A}_{3} \tilde{\mathbf{q}}-\Delta \mathbf{d}=\mathbf{v} .
$$

Equation (32) says that the mean values of the orbit elements and the mean values of the pseudo-stochastic pulses may be estimated from (34) only with a poor quality (in the approximation (32) this would not be possible at all), whereas the difference between the orbital elements and the pulses may be established rather accurately.

Equations (25) and (34) are algebraically equivalent. From the numerical perspective version (34) is much better, because it separates those satellite-specific parameters, which may be established well (and do not pose any problems in a preelimination procedure) from those, which are poorly determined by the K-Band observations (and which might be put to zero in a first approximation).

Based on the observation equations (34) the following modified NEQ contribution of K-Band observations may be set up:

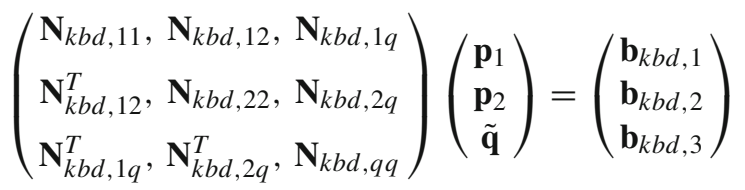

The parameter transformation (33) also has to be applied to the GPS part (23) of the analysis, because we have to stack the NEQ contributions stemming from GPS and the contribution 
stemming from the K-Band. The combined NEQ contribution from GPS before performing the parameter transformation simply reads as:

$$
\left(\begin{array}{ccc}
\mathbf{N}_{11}, & \mathbf{0}, & \mathbf{N}_{1 q} \\
\mathbf{0}, & \mathbf{M}_{22}, & \mathbf{M}_{2 q} \\
\mathbf{N}_{1 q}^{T}, & \mathbf{M}_{2 q}^{T}, & \mathbf{N}_{q q}+\mathbf{M}_{q q}
\end{array}\right)\left(\begin{array}{c}
\mathbf{o}_{1} \\
\mathbf{o}_{2} \\
\mathbf{q}
\end{array}\right)=\left(\begin{array}{c}
\mathbf{b}_{1} \\
\mathbf{c}_{2} \\
\mathbf{b}_{q}+\mathbf{c}_{q}
\end{array}\right) .
$$

By applying the parameter transformation (33) to the above combined equation one obtains:

$$
\begin{aligned}
& \left(\begin{array}{lll}
\mathbf{N}_{11}+\mathbf{M}_{22}, & \mathbf{N}_{11}-\mathbf{M}_{22}, & \mathbf{N}_{1 q}+\mathbf{M}_{2 q} \\
\mathbf{N}_{11}^{T}-\mathbf{M}_{22}^{T}, & \mathbf{N}_{11}+\mathbf{M}_{22}, & \mathbf{N}_{1 q}-\mathbf{M}_{2 q} \\
\mathbf{N}_{1 q}^{T}+\mathbf{M}_{2 q}^{T}, & \mathbf{N}_{1 q}^{T}-\mathbf{M}_{2 q}^{T}, & \mathbf{N}_{q q}+\mathbf{M}_{q q}
\end{array}\right)\left(\begin{array}{c}
\mathbf{p}_{1} \\
\mathbf{p}_{2} \\
\mathbf{q}
\end{array}\right) \\
& =\left(\begin{array}{c}
\mathbf{b}_{1}+\mathbf{c}_{2} \\
\mathbf{b}_{1}-\mathbf{c}_{2} \\
\mathbf{b}_{q}+\mathbf{c}_{q}
\end{array}\right) .
\end{aligned}
$$

Using the same kind of arguments as in the case of the K-Band equations, we conclude that the parameters $\mathbf{p}_{1}$ are better established than the $\mathbf{p}_{2}$. The superposition of the transformed K-Band NEQ contribution (35) and the combined and transformed GPS-contribution (37) (with appropriate weights) should therefore ideally strengthen the determination of both parameter types.

The transformation greatly reduces the loss of significant digits of the combined GPS and K-Band solution. It is, however, even more important that different constraints may be imposed on the mean values and half-differences of the pseudo-stochastic parameters. To combine the daily GPS- and K-Band-files into one daily file containing both contributions one still has to weight the K-Band solution with $\sigma_{\mathrm{ph}}^{2} / \sigma_{k b d}^{2}$, but the effect is numerically much less detrimental, because the terms $\mathbf{N}_{11}+\mathbf{M}_{22}$ remain large in absolute value, whereas the corresponding term $\mathbf{N}_{k b d, 11}$ in the K-Band Eq. (35) becomes very small; the terms $\mathbf{N}_{11}-\mathbf{M}_{22}$, on the other hand, are becoming small in absolute value and hardly influence the term $\mathbf{N}_{k b d, 22}$ when superposing Eqs. (35) and (37).

\subsection{Orbit determination}

Orbit determination in its simplest form was defined in Sect. 2.7 as the task of estimating six parameters defining the initial state vector (plus optionally a certain number of satellite- and arc-specific parameters, e.g., pulses) from a set of observations. In the context of gravity field determination using satellites and/or satellite constellations orbit determination serves two purposes in the CMA:

1. The generation of a priori orbits (reference orbits) $\mathbf{r}_{0}(t)$ based on all observations (GPS and K-Band) within the arc.
2. The generation of the residuals for all observations after gravity field determination.

The first task was already discussed in Sect. 3.5. The solution of the second task is actually almost identical with the solution of the first one when using the estimated gravity field as the a priori gravity field. The only difference concerns step four of the procedure: the parametrization will not be changed to contain the gravity field parameters. The resulting standard orbits may now be used to generate the residuals of the gravity field determination process. It is important, in particular when using kinematic positions and inter-satellite measurements for gravity field determination, to generate the residuals for all observation types.

\subsection{Empirical and non-gravitational forces}

In Sect. 2.8 the distinction was made of methods based uniquely on the Class I observations and those using in addition Class II observations (in particular the accelerometer data). Both options are available in the CMA. One may easily switch from one mode to the other if the empirical dynamic parameters are the same in both cases. When, e.g., setting up constant and once-per-revolution terms for the three components $R, S$, and $W$-and solving for the corresponding scaling parameters-these empirical models may be interpreted as bias parameters for the accelerometer data. When not using the accelerometer data the same terms absorb to the extent possible also the deterministic part describing the non-gravitational forces. The only difference between the two options to deal with the non-gravitational forces resides in the constraints, which are put on the pulses (accelerations). Whereas these constraints have to be relatively loose when not using the accelerometer data, they may be rather tight when making use of the accelerometer data. The concrete settings of the constraints are an important issue of the analysis. Needless to say that the gravity field results improve, if the constraints can be tightened - as long as the non-gravitational contributions still may be absorbed by the empirical terms. The issue is further studied by Beutler et al. (2010).

\section{Methods for gravity field recovery using GRACE}

Many methods for determining static and time varying gravity fields were developed and applied to GRACE data in recent years. To point out a few important differences between the CMA and alternative approaches, we briefly discuss four of them, namely the developments of the

1. GeoForschungszzentrum Potsdam/Groupe de Recherche de Géodésie Spatiale (Potsdam/Toulouse), resulting in the series of the EIGEN gravity fields (Förste et al. 2008), 
2. Center for Space Research (University of Texas at Austin), resulting in the series of GGM gravity fields (Tapley et al. 2005),

3. Institut für Geodäsie und Geoinformation in Bonn, resulting in the series of ITG gravity fields (Mayer-Gürr 2008),

4. Delft Institute of Earth Observation and Space Systems (DEOS) resulting in the first release of DEOS Mass Transport (DMT-1) monthly models (Liu 2008).

The first two methods are classical dynamic orbit and gravity adjustment processes, which have their roots in the analysis of SLR observations. The two methods are similar from the point of view of structure and parametrization, they differ somewhat in the establishment of the GPS contribution: the EIGEN solutions first determine the GPS orbits and GPS clock corrections using data from a ground tracking network, then the GPS-derived NEQs for orbit and gravity field determination are exploited using the LEO/GPS data together with the GPS orbits and clocks determined previously. The GGM solutions rely on the GPS orbits and GPS clock corrections from the IGS. The GPS-specific NEQs for LEO orbit determination and gravity field determination are then established as in the EIGEN solutions. The K-Band range-rates are analyzed in the same way in both solutions: the accelerometer offset parameters are set up in the three orthogonal directions for each day, one accelerometer scale is estimated for each day in the case of the EIGEN solutions, one per month for the GGM solutions. No attempt is made to absorb the deficiencies of the force field model by setting up other than the accelerometer-related empirical dynamic parameters in the adjustment process. In order to absorb such effects and possible errors in the K-Band range-rate observable, so-called K-Band empirical parameters (also called kinematic parameters), namely, biases, slopes, and once-per-revolution parameters are introduced. One set of such parameters is set up every $45 \mathrm{~min}$ for biases and slopes, every $90 \mathrm{~min}$ for the onceper-rev terms. The parametrization follows in essence the recommendations made by Kim (2000) and obviously tries to avoid the absorption of gravity field signals by empirical dynamic parameters. The GPS- and K-Band NEQs are combined in the same way as in the CMA. The main differences to the CMA are (a) the absence of pseudo-stochastic parameters, (b) the use of empirical parameters for K-Band, and (c) the use of the original GPS observables instead of the kinematic positions.

The procedures for gravity field determination developed by Mayer-Gürr (2008) differ from the aforementioned methods by (a) using the positions from the GRACE science orbit as kinematic positions, by (b) using short-arcs of $30 \mathrm{~min}$, by (c) using the K-Band ranges as the principal observations, and by (d) taking the impact of accelerometer noise on the K-Band observable into account through a covariance matrix. The model used by Mayer-Gürr (2008) is closely related to setting up pulses frequently. We use Eqs. (30) as the starting point to demonstrate this relationship. The equations are modified to contain the pulses related to the two satellites in array $\mathbf{p}$, the other orbit parameters and the gravity field parameters in array $\mathbf{q}^{\prime}$ :

$\tilde{\mathbf{A}}_{q}^{\prime} \tilde{\mathbf{q}}^{\prime}+\tilde{\mathbf{A}}_{p} \mathbf{p}-\Delta \mathbf{d}=\mathbf{v}$.

According to Sect. 3.9 the constraints put on the pulses are captured by the following pseudo-observation equations:

$\mathbf{p}-\mathbf{0}=\mathbf{v}_{p}$,

where $\mathbf{v}_{p}$ are the residuals associated with the pulses (in this special case identical with the pulses). When assigning the weight of 1 to the range observations (assumed as uncorrelated with an RMS error of $\sigma_{k b d}$ ) and the weight $\sigma_{k b d}^{2} / \sigma_{p}^{2}$ to the pulses, the following NEQ results from Eqs. $(38,39)$ :

$$
\left(\begin{array}{cc}
\tilde{\mathbf{A}}_{q}^{\prime T} \tilde{\mathbf{A}}_{q}^{\prime}, & \tilde{\mathbf{A}}_{q}^{\prime T} \tilde{\mathbf{A}}_{p} \\
\tilde{\mathbf{A}}_{p}^{T} \tilde{\mathbf{A}}_{q}^{\prime}, & \tilde{\mathbf{A}}_{p}^{T} \tilde{\mathbf{A}}_{p}+\frac{\sigma_{k b d}^{2}}{\sigma_{p}^{2}} \mathbf{U}_{p}
\end{array}\right)\left(\begin{array}{c}
\tilde{\mathbf{q}}^{\prime} \\
\mathbf{p}
\end{array}\right)=\left(\begin{array}{c}
\tilde{\mathbf{A}}_{q}^{\prime T} \Delta \mathbf{d} \\
\tilde{\mathbf{A}}_{p}^{T} \Delta \mathbf{d}
\end{array}\right),
$$

where $\mathbf{U}_{p}$ is the identity matrix (the dimension of which equals the number of pulses). If the pulses are not of interest and if the GPS NEQs are not considered, the vector $\mathbf{p}$ may be pre-eliminated in the NEQs (35). The result may by written as:

$\tilde{\mathbf{A}}_{q}^{\prime T} \mathbf{P} \tilde{\mathbf{A}}_{q}^{\prime} \tilde{\mathbf{q}}^{\prime}=\tilde{\mathbf{A}}_{q}^{\prime T} \mathbf{P} \Delta \mathbf{d}$,

with

$\mathbf{P}=\mathbf{U}_{q^{\prime}}-\frac{\sigma_{p}^{2}}{\sigma_{k b d}^{2}} \tilde{\mathbf{A}}_{p}\left\{\mathbf{U}_{p}+\frac{\sigma_{p}^{2}}{\sigma_{k b d}^{2}} \tilde{\mathbf{A}}_{p}^{T} \tilde{\mathbf{A}}_{p}\right\}^{-1} \tilde{\mathbf{A}}_{p}^{T}$.

Equation (42) is identical with the weight matrix resulting from the covariance matrix (4.193) proposed by Mayer-Gürr (2008) for the range observable (when assuming uncorrelated ranges, uncorrelated pulses, and using the same approximation as above). The statistical model used by Mayer-Gürr (2008) for short-arcs is thus very closely related to our pseudo-stochastic model emerging from constrained pulses.

The above developments neglect the impact of the pulses on the GPS observation equations. This neglect is not important for short-arcs, because the impact of orbit deficiencies on the GPS observation equations is greatly reduced by the classical orbit parameters of the short-arcs (initial or boundary values) and because the impact of the constrained pulses within the short-arc on the kinematic positions is well below their RMS errors. Equation (42) should not be used for longarcs. Formulas analogous to Eq. (35) have to be set up for the GPS contributions of GRACE-A and -B and these have 
to be superposed to Eq. (35) using the correct weight ratio prior to the pre-elimination of the pulses.

Let us conclude this section with a few comments concerning a class of methods determining the gravity field without including orbit parameters in the estimation process. The socalled acceleration approach, applied to observations from a single satellite, is used as an example, because it is very transparent. One of the latest treatments of this method and its application to GRACE data is due to Liu (2008).

We assume that the second derivatives of the position vector have been observed (or derived by numerical differentiation from kinematic positions). The CMA observation equations for the accelerations referring to epoch $t_{l}$ follow from Eq. (20) by taking twice the time derivative at $t_{l}$ :

$$
\sum_{k=1}^{6+3 n_{s}} \frac{\partial \ddot{\mathbf{r}}\left(t_{l}\right)}{\partial o_{k}} \cdot o_{k}+\sum_{k=1}^{d} \frac{\partial \ddot{\mathbf{r}}\left(t_{l}\right)}{\partial q_{k}} \cdot q_{k}-\Delta \ddot{\mathbf{r}}_{l}=\tilde{\mathbf{v}}_{l},
$$

where it was assumed that no arc-specific empirical dynamic parameters were set up. $\Delta \ddot{\mathbf{r}}_{l}$ stands for the observed minus the computed acceleration. The partial derivatives in the second sum may be replaced by the right-hand sides of the variational equations (14):

$$
\frac{\partial \ddot{\mathbf{r}}\left(t_{l}\right)}{\partial q_{k}}=\frac{\partial \mathbf{f}\left(t_{l}\right)}{\partial \mathbf{r}\left(t_{l}\right)} \cdot \frac{\partial \mathbf{r}\left(t_{l}\right)}{\partial q_{k}}+\frac{\partial \mathbf{f}\left(t_{l}\right)}{\partial \dot{\mathbf{r}}\left(t_{l}\right)} \cdot \frac{\partial \dot{\mathbf{r}}\left(t_{l}\right)}{\partial q_{k}}+\frac{\partial \mathbf{f}\left(t_{l}\right)}{\partial q_{k}},
$$

where use was made of Eq. (15). The observation equations used in the acceleration approach simply read as:

$$
\sum_{k=1}^{d} \frac{\partial \mathbf{f}\left(t_{l}\right)}{\partial q_{k}} \cdot q_{k}-\Delta \ddot{\mathbf{r}}_{l}=\mathbf{v}_{l} .
$$

When using an a priori orbit solving the equations of motion parameterized with the parameters $o_{k}, k=1,2, \ldots, 6+3 n_{s}$, Eq. (45) implies from the standpoint of the CMA that the following approximation was made:

$$
\begin{aligned}
& \sum_{k=1}^{6+3 n_{s}} \frac{\partial \ddot{\mathbf{r}}\left(t_{l}\right)}{\partial o_{k}} \cdot o_{k}+\sum_{k=1}^{d}\left[\frac{\partial \mathbf{f}\left(t_{l}\right)}{\partial \mathbf{r}\left(t_{l}\right)} \cdot \frac{\partial \mathbf{r}\left(t_{l}\right)}{\partial q_{k}}+\frac{\partial \mathbf{f}\left(t_{l}\right)}{\partial \dot{\mathbf{r}}\left(t_{l}\right)} \cdot \frac{\partial \dot{\mathbf{r}}\left(t_{l}\right)}{\partial q_{k}}\right] \\
& \quad q_{k} \doteq \mathbf{0} .
\end{aligned}
$$

The acceleration method thus assumes that the changes in the second derivatives of the orbit caused by the estimated gravity field parameters $q_{k}$ are counterbalanced by changes of the second derivatives of the orbit due to the changes in the arc-specific parameters $o_{k}$. The assumption is obviously met if the a priori orbit used to compute $\Delta \ddot{\mathbf{r}}_{l}$ in the acceleration approach equals the estimated a posteriori orbit resulting in the CMA. If this is not the case, the assumption cannot be met precisely. The actual error introduced into the observation equations of the acceleration method on top of the inevitable linearization error depends on the quality of the a priori orbit. The method is successfully used to analyze CHAMP and GRACE data by Liu (2008) and others, indicating that the neglect may be acceptably small for certain applications.

The acceleration approach was developed "...to avoid costly computations employed in conventional techniques (e.g., computing the partial derivatives which require a numerical integration of variation equations) ..." Liu (2008). In Sect. 3.7 it was, however, shown that the solution of the variational equations, as performed in the CMA, is neither time consuming nor costly for dynamical parameters including gravity field parameters.

\section{Summary and conclusions}

The key issues of celestial mechanics related to orbit and gravity field estimation were outlined in Sect. 2, the specific characteristics of the CMA and its responses to the mentioned issues of celestial mechanics were developed in Sect. 3.

The CMA is designed for orbit and gravity field determination of constellations of satellites using kinematic positions determined by GPS as pseudo-observations and ultra-precise inter-satellite measurements.

Gravity field coefficients, range biases, initial osculating elements, empirical dynamic parameters, accelerometerspecific parameters, regularly spaced pseudo-stochastic parameters, either pulses or constant accelerations, may be set up in the $R, S$, and $W$ directions. The latter parameters may be constrained.

The variational equations associated with each parameter are second-order linear differential equations, which are solved very efficiently in the CMA: the solutions corresponding to pseudo-stochastic pulses are obtained as linear combinations of the solutions of the six variational equations associated with the initial osculating elements. The coefficients of the linear combination for each pulse are constant and are derived from a system of six linear equations. The solutions of all other variational equations (associated with the gravity field, the accelerometers, and empirical dynamic parameters) are also obtained as linear combinations of the solutions of the six variational equations associated with the six initial osculating elements, but the coefficients are functions of time. The coefficients are obtained by numerical quadrature. The time (CPU) required to solve the variational equations is not an important issue in the CMA.

In order to allow for maximum flexibility all satellitespecific parameters $\mathbf{0}_{1}$ and $\mathbf{o}_{2}$ are transformed according to Eq. (33) to give the mean value $\mathbf{p}_{1}$ and half of their difference $\mathbf{p}_{2}$ as a result.

Figure 1 in Sect. 2 shows that even very small, normally distributed stochastic variations of the acceleration field may have a seizable influence on the orbits, thus also on the measured inter-satellite distances and therefore should be considered together with the noise characteristics of the other 
measurements (range and GPS-derived observations). Note, that the experiment documented by Fig. 1 was based, as stated in Sect. 2, on simplifying assumptions. An in-depth analysis should not be based simply on a comparison of RMS values of the involved observations, because of a possible dependency of noise on frequency. Such an analysis should include the power spectral densities of the range (or the range-rate), the accelerometer, and the GPS-related observables.

In the CMA the perturbations with periods longer than the spacing between the pseudo-stochastic parameters are to a great extent absorbed by the pseudo-stochastic parameters, either pulses of piece-wise constant accelerations. Beutler et al. (2010) illustrate many of the key components of the CMA using a limited amount of data of the GRACE mission.

Acknowledgments This work was supported by the Swiss National Science Foundation (project "gravity field determination using positions of low Earth orbiters established with the GPS" with number 200020117908) and by the Institute for Advanced study (IAS) of the Technische Universität München. Both contributions are gratefully acknowledged.

\section{References}

Altamimi Z, Collilieux X, Legrand J, Garayt B, Boucher C (2007) ITRF2005: a new release of the international terrestrial reference frame based on time series of station positions and Earth orientation parameters. J Geophys Res 112:B09401. doi:10.1029/ 2007JB004949

Beutler G, Brockmann E, Gurtner W, Hugentobler U, Mervart L, Rothacher M (1994) Extended orbit modeling techniques at the CODE processing center of the international GPS service for geodynamics (IGS): theory and initial results. Manuscr Geod 19:367386

Beutler G (2005) Methods of celestial mechanics. Springer, Berlin

Beutler G, Jäggi A, Hugentobler U, Mervart L (2006) Efficient satellite orbit modelling using pseudo-stochastic parameters. J Geod 80(7): 353-372. doi:10.1007/s00190-006-0072-6

Beutler G, Jäggi A, Meyer U, Mervart L (2010) The celestial mechanics approach: application to data of the GRACE mission. J Geod. doi:10.1007/s00190-010-0402-6

Biancale R, Balmino G, Lemoine J-M, Marty J-C, Moynot B, Barlier F, Exertier P, Laurain O, Gegout P, Schwintzer P, Reigber C, Bode A, König R, Massmann F-H, Raimondo J-C, Schmidt R, Zhu SY (2000) A new global Earth's gravity field model from satellite orbit perturbations: GRIM5-S1. Geophys Res Lett 27(22):36113614. doi:10.1029/2000GL011721

Dach R, Hugentobler U, Meindl M, Fridez P (eds) (2007) The Bernese GPS Software Version 5.0. Astronomical Institute, University of Bern

Drinkwater M, Haagmans R, Muzi D, Popescu A, Floberghagen R, Kern M, Fehringer M (2006) The GOCE gravity mission: ESA's first core explorer. ESA SP-627, ESA Publication Division, pp 1-7

Flechtner F (2005) AOD1B product description document. Technical Report GRACE 327-750, JPL. http://podaac.jpl.nasa.gov/pub/ grace/doc/newsletters/GRACE_SDS_NL_0401.pdf

Förste C, Schmidt R, Stubenvoll R, Flechtner F, Meyer U, König R, Meumayer H, Biancale R, Lemoine JM, Bruinsma S, Loyer S, Barthelmes F, Esselborn S (2008) The GeoForschungszentrum Potsdam/Groupe de Recherche de Géodésie Spatiale satellite-only and combined gravity field models: EIGEN-GL04S1 and EIGENGL04C. J Geod 82(6):331-346. doi:10.1007/s00190-007-0183-8
Jäggi A, Beutler G, Hugentobler U (2006) Pseudo-stochastic orbit modeling techniques for low-Earth orbiters. J Geod 80(1):47-60. doi:10.1007/s00190-006-0029-9

Jäggi A (2007) Pseudo-stochastic orbit modeling of low Earth satellites using the Global Positioning System. Geodätisch-geophysikalische Arbeiten in der Schweiz, vol 73, Schweizerische Geodätische Kommission, Institut für Geodäsie und Photogrammetrie, Eidg. Technische Hochschule zürich, zürich

Jäggi A, Dach R, Montenbruck O, Hugentobler U, Bock H, Beutler G (2009a) Phase center modeling for LEO GPS receiver antennas and its impact on precise orbit determination. J Geod 83(12):11451162. doi:10.1007/s00190-009-0333-2

Jäggi A, Beutler G, Prange L, Meyer U, Mervart L, Dach R, Rummel R, Gruber T (2009b) Gravity field determination at AIUB: current activities. EGU General Assembly 2009, EGU2009-8714

Jäggi A, Beutler G, Mervart L (2010a) GRACE gravity field determination using the celestial mechanics approach-first results. In: Mertikas S (ed) Gravity, geoid and Earth observation. Springer, Berlin, pp 177-184. doi:10.1007/978-3-642-10634-7-24

Jäggi A, Beutler G, Meyer U, Mervart L, Prange L, Dach R (2010b) AIUB-GRACE02S - status of GRACE gravity field recovery using the celestial mechanics approach. In: International association of geodesy symposium (accepted)

Kim J (2000) Simulation study of a low-low satellite-to-satellite tracking mission. PhD Dissertation, The University of Texas at Austin

Lemoine FG, Smith DE, Kunz L, Smith R, Pavlis NK, Klosko SM, Chinn DS, Torrence MH, Willamson RG, Cox CM, Rachlin KE, Wang YM, Kenyon SC, Salman R, Trimmer R, Rapp RH, Nerem RS (1997) The development of the NASA GSFC and NIMA joint geopotential model. In: Segawa J, Fujimoto H, Okubo S (eds) IAG symposia: gravity, geoid and marine geodesy. Springer, Berlin, pp 461-469

Liu X (2008) Global gravity field recovery from satallite-to-satellite tracking data with the acceleration approach. Publications on Geodesy, Nederlandse Commissie voor Geodesie, Netherlands Geodetic Commission, No 68

Liu X, Ditmar P, Siemes C, Slobbe DC, Revtova E, Klees R, Riva R, Zhao Q (2010) DEOS mass transport model (DMT-1) based on GRACE satellite data: methodology and validation. Geophys J Int. doi:10.1111/j.1365-246X.2010.04533.x

Lyard F, Lefevre F, Letellier T, Francis O (2006) Modelling the global ocean tides: insight from FES2004. Ocean Dyn 56:394-415

Mayer-Gürr T, Ilk KH, Eicker A, Feuchtinger M (2005) ITGCHAMP01: a CHAMP gravity field model from short kinematical arcs of a one-year observation period. J Geod 78:462-480

Mayer-Gürr T (2008) Gravitationsfeldbestimmung aus der Analyse kurzer Bahnbögen am Beispiel der Satellitenmissionen CHAMP und GRACE. Dissertation Schriftenreihe Institut für Geodäsie und Geoinformation No 9, University of Bonn

McCarthy DD, Petit G (eds) (2003) IERS conventions (2003), international Earth rotation and reference systems service (IERS). IERS Technical Note No. 32, Verlag des Bundesamtes für Karthographie und Geodäsie, Frankfurt am Main, 2004

Montenbruck O, Garcia-Fernandez M, Williams J (2006) Performance comparison of semi-codeless GPS receivers for LEO satellites. GPS Solut 10(4):249-261. doi:10.1007/s10291-006-0025-9

Pail R, Metzler B, Lackner B, Preimesberger T, Höck E, Schuh WD, Alkathib H, Boxhammer C, Siemes C, Wermuth M (2006) GOCE gravity field analysis in the framework of HPF: operational softwaresystem and simulation results. In: 3rd GOCE User workshop, Frascati, Italy, ESA SP-627, pp 249-256, 6-8 November 2006

Prange L, Jäggi A, Beutler G, Mervart L, Dach R (2009) Gravity field determination at the AIUB - the celestial mechanics approach. In: Sideris MG (ed) observing our changing Earth. Springer, Berlin, pp 353-362. doi:10.1007/978-3-540-85426-5-42 
Prange L, Jäggi A, Bock H, Dach R (2010) The AIUB-CHAMP02S and the influence of GNSS model changes on gravity field recovery using spaceborne GPS. Adv Space Res 45(2):215-224. doi:10. 1016/j.asr.2009.09.020

Reigber C, Jochmann H, Wünsch J, Petrovic S, Schwintzer F, Barthelmes F, Neumayer K H, König R, Förste C, Balmino G, Biancale R, Lemoine JM, Loyer S, Pérosanz F (2004) Earth gravity field and seasonal variability from CHAMP. In: Reigber C, Schwintzer P, Wickert J (eds) Earth observation from CHAMPresults from three years in orbit. Springer, Berlin, pp 25-30

Seidelmann PK (ed) (1992) Explanatory supplement to the astronomical almanac. University Science Books, Mill Valley

Strang G, Borre K (1997) Linear algebra, geodesy, and GPS. Wellesley-Cambridge Press, Wellesley

Švehla D, Rothacher M (2004) Kinematic precise orbit determination for gravity field determination. In: Sansò $\mathrm{F}$ (ed) A window on the future of geodesy. Springer, Berlin, pp 181-188. doi:10.1007/ 3-540-27432-4-32
Tapley BD, Bettadpur S, Ries JC, Thompson PF, Watkins M (2004) GRACE measurements of mass variability in the Earth system. Science 305(5683): 503-505

Tapley BD, Ries J, Bettapour S, Chambers D, Cheng M, Condi F, Gunter B, Kang Z, Nagel P, Pastor R, Pekker T, Poole S, Wang F (2005) GGM02-an improved Earth gravity model from GRACE. J Geod 79:467-478

Thomas JB (1999) An analysis of gravity-field estimation based on intersatellite dual-1-way biased ranging JPL Publication 98-15. http://podaac.jpl.nasa.gov/pub/grace/doc/newsletters/GRACE_ SDS_NL_0401.pdf

Touboul P, Willemenot E, Foulon B, Josselin V (1999) Accelerometers for CHAMP, GRACE and GOCE space missions: synergy and evolution. Boll Geofis Teor Appl 40:321-327

Zumberge JF, Heflin MB, Jefferson DC, Watkins MM, Webb FH (1997) Precise point positioning for the efficient and robust analysis of GPS data from large networks. J Geophys Res 102:5005-5017 